\title{
Parasite communities indicate effects of cross-shelf distributions, but not mesoscale oceanographic features on northern California Current mid-trophic food web
}

\author{
Kym C. Jacobson ${ }^{1, *}$, Rebecca Baldwin ${ }^{2}$, Douglas C. Reese ${ }^{3}$ \\ ${ }^{1}$ Newport Field Station, Northwest Fisheries Science Center, National Marine Fisheries Service, \\ National Oceanographic and Atmospheric Administration, 2030 S.E. Marine Science Dr., Newport, Oregon 97365, USA \\ ${ }^{2}$ Department of Biological Sciences, University of Alberta, Edmonton, Alberta, T6G 2E9, Canada \\ ${ }^{3}$ Department of Fisheries and Wildlife, Oregon State University, Corvallis, Oregon, 97331, USA
}

\begin{abstract}
Mesoscale physical oceanographic features, such as jets and eddies, can influence the structure of marine ecosystems. We used trophically transmitted parasite communities of pelagic fishes in the northern California Current to examine effects of physical oceanographic features on pelagic ecosystem structure. We tested the hypotheses that (1) oceanographic features associated with a coastal promontory, Cape Blanco, Oregon (USA), produced a faunal break resulting in different pelagic ecosystems north and south of the cape, and that (2) the use of biological hotspots in the area by pelagic nekton is reflected in the trophic interactions of mid- and upper trophic level fishes. We recovered 19 taxa of trophically transmitted parasites from $10 \mathrm{com}-$ mon pelagic fish species caught between Newport, Oregon, and Crescent City, California. Nonmetric multidimensional scaling of parasite communities reflected a trophic structure among these fish species; results were similar to published diet studies. We found no evidence in the trophically transmitted parasites of spatial differences between the pelagic ecosystems north or south of Cape Blanco, or within versus outside of the biological hotspots. However, we found significant cross shelf differences in parasite communities. Therefore, Cape Blanco does not seem to be a strong faunal boundary, rather the strongest influence is cross-shelf transport associated with coastal upwelling.
\end{abstract}

KEY WORDS: Marine parasites · Pelagic fish $\cdot$ Cape Blanco $\cdot$ Hotspot $\cdot$ Inshore-offshore

Resale or republication not permitted without written consent of the publisher

\section{INTRODUCTION}

The California Current System (CCS) is 1 of the world's 4 large Eastern Boundary current upwelling systems: Canary, Peru, Benguela, and California. These highly productive upwelling ecosystems produce one-fifth of the marine fish harvested globally (Fréon et al. 2009). In the Northeast Pacific, the CCS supports valuable fisheries for both highly migratory species such as Pacific hake Merluccius productus (Bailey et al. 1982) and Pacific sardine
Sardinops sagax (reviewed by Checkley et al. 2009), and less migratory small pelagic species such as Pacific herring Clupea pallasii (Flostrand et al. 2009) and northern anchovy Engraulis mordax (reviewed by Checkley et al. 2009). It also supports many species of economically and culturally important salmon, including coho Oncorhynchus kisutch and Chinook O. tshawytscha salmon, as well as economically important crustaceans such as Dungeness crab Cancer magister and pink shrimp Pandalus jordani. 
The productivity of the northern California Current (NCC) region is driven largely by large-scale climatic forcing through wind-driven upwelling and shifts in the subarctic gyre that bring arctic or subtropical waters to the Washington and Oregon shelf (Keister et al. 2011). However, topography at local scales (e.g. submarine banks, canyons) can also influence upwelling regions. In addition, major promontories along the US west coast result in differences in physical and biological characteristics within the CCS (Batchelder et al. 2002). Cape Blanco, a promontory on the southern coast of Oregon $\left(43^{\circ} \mathrm{N}\right.$; Fig. 1), has been reported to generate mesoscale physical oceanographic features, including a separating coastal upwelling jet (Barth et al. 2000) that affects the advection and retention of zooplankton and secondary local production as well as ecosystem structure (Batchelder et al. 2002, Keister et al. 2009).

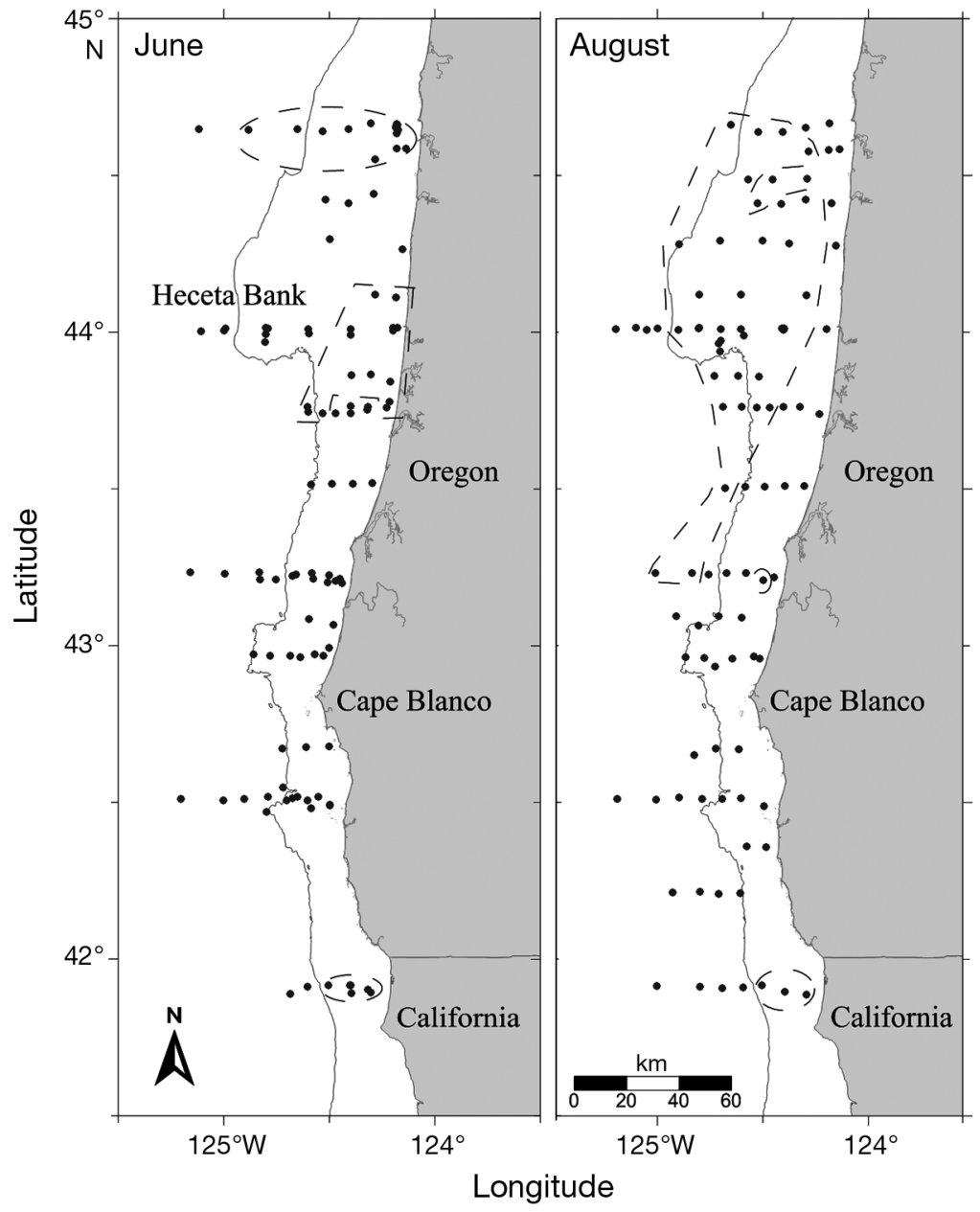

Fig. 1. Sampling stations $(\bullet)$ for the US GLOBEC Northeast Pacific Program in 2002. Transects were situated east to west. Solid line $=200 \mathrm{~m}$ isobath. Stations identified by Reese \& Brodeur (2006) to be in a hotspot are enclosed in dashed lines
To the north of Cape Blanco, the topography of Heceta Bank, a substantial shelf-bank at 44 to $44.4^{\circ} \mathrm{N}$, has also been proposed to interact with the summer coastal jet and affect ecosystem structure (Batchelder et al. 2002).

Biological hotspots are regions of high productivity and are often associated with mesoscale features. These areas of high regional productivity have been identified as areas of high chlorophyll concentration (Valavanis et al. 2004) and as geographic areas with increased diversity and abundance of fish species (Reese \& Brodeur 2006). Species from multiple trophic levels may be attracted to oceanographic hotspots for foraging (e.g. Piatt et al. 2006, Polovina et al. 2006, Sydeman et al. 2006).

The importance of mesoscale features to fishes is often assessed by increased abundance of enhanced feeding (shown through stomach content analysis). We present a unique way to investigate the response of fishes to mesoscale features, through examination of the parasite communities acquired through trophic interactions. The transmission of trophically transmitted parasites is sensitive to the physical environment as well as to predator-prey interactions, and thus they can be used to better understand both physical and biotic drivers of ecosystem structure. Many marine parasites have free-living stages that hatch from eggs and are subject to the same oceanographic physical conditions (e.g. temperature, salinity, currents) that affect hatching and recruitment of larval stages and distributions of other marine organisms (reviewed by Marcogliese 2005). Furthermore, the distributions and abundances of zooplankton, which serve as intermediate hosts (required for parasite development) for many marine parasites, also affect the transmission, abundance, and thus community structure of trophically transmitted parasites in marine fish (Marcogliese 1995). Once a fish has acquired a parasite through feeding either directly on zooplankton or on a higher trophic-level fish that feeds on zooplankton, the parasites can remain in the body from months to years, depending upon the host-parasite 
association (Rohde 1984), and can accumulate over time. The longevity of marine parasites in fish (Campbell 1983) can provide an extended history of trophic interactions beyond the period reflected by stomach contents (Klimpel et al. 2003, Baldwin et al. 2008, Valtonen et al. 2010).

This study was designed to use trophically transmitted parasites to address a hypothesis of the United States Global Ocean Ecosystem Dynamics (US GLOBEC) Northeast Pacific Program: that spatial and temporal variability in mesoscale circulation constitute the dominant physical forces that structure variations in zooplankton biomass, production, distribution, and species interactions in coastal regions (Strub et al. 2002). Target species identified in the program included copepods, euphausiids, juvenile coho and Chinook salmon, and their predators. Process studies were geographically focused on Heceta Bank (an area of retention) and Cape Blanco (hypothesized to be a faunal boundary) to gain a better understanding of the biophysical mechanisms that link zooplankton and salmon populations to their physical environment (Batchelder et al. 2002). Here we examined the trophically transmitted parasite communities of juvenile salmon and other pelagic fish species in the NCC, firstly to examine how well the trophically transmitted parasites reflected the mid-trophic food web, and secondly to test the hypothesis that oceanographic mesoscale features (Cape Blanco and biological hot spots) that affect zooplankton distributions also affect the mid-trophic food web as indexed by parasites.

\section{MATERIALS AND METHODS}

\section{Study area}

Sampling was conducted during 2002 (Fig. 1) as part of the US GLOBEC Northeast Pacific Program (Brodeur et al. 2004, Reese \& Brodeur 2006). The study area is a $300 \mathrm{~km}$ region extending from Newport, Oregon $\left(44^{\circ} 39^{\prime} \mathrm{N}\right)$, to Crescent City, California $\left(41^{\circ} 54^{\prime} \mathrm{N}\right)$. Fishes included in these analyses were collected from 31 May to 18 June 2002 (hereafter referred to as the June cruise) and 1 August to $18 \mathrm{Au}-$ gust 2002 (August cruise). The August field effort was included to capture any effects of a fully developed late-summer circulation pattern in the CCS (Barth et al. 2000). Fish were collected at predetermined stations (Fig. 1) selected from long-term observation programs (Batchelder et al. 2002). Opportunistic collections were also made in areas of biological or oceanographic interest. Water temperature, salinity, depth, and distance offshore were measured at each station (Brodeur et al. 2004). Stations between the shore and the $200 \mathrm{~m}$ isobaths (representing the continental shelf break) were defined as inshore, and stations beyond this line were designated as offshore.

\section{Fish and macroparasite collections}

Fish were collected with a 264 rope trawl (Nor'Eastern Trawl Systems) that has a 30 m wide by $20 \mathrm{~m}$ deep mouth opening and variable mesh sizes (1626 $\mathrm{mm}$ at mouth to $89 \mathrm{~mm}$ at cod end). To maintain catches of small fish and squid, a $6.1 \mathrm{~m}$ long, $0.8 \mathrm{~cm}$ mesh knotless liner was sewn into the cod end. Trawling was performed for a maximum of $30 \mathrm{~min}$ per station at a towing speed of $6 \mathrm{~km} \mathrm{~h}^{-1}$. Fish were identified at sea, fork length (FL) was measured to the nearest $\mathrm{mm}$, and a portion of the catch was then immediately frozen for later necropsy. Ten pelagic fish species, totaling 1203 individual fish, were analyzed for trophically transmitted parasites. These included juvenile and subadult Chinook salmon and juvenile coho salmon; small pelagic planktivorous fish ('forage fish'), viz. northern anchovy, Pacific herring, Pacific sardine, Pacific saury Cololabis saira, surf smelt Hypomesus pretiosus, and whitebait smelt Allosmerus elongatus; and large pelagics (planktivorous as juveniles, piscivorous as adults), i.e. jack mackerel Trachurus symmetricus and Pacific hake (Emmett \& Krutzikowsky 2008). Due to the variation in FL of juvenile salmon, Chinook salmon were classified as either yearlings or subadults based on FL (Brodeur et al. 2004). Too few subadult coho salmon were caught to be included in this study. Based on frequency distributions of FL (data not shown), all other fish included in this study were identified as adults.

In the laboratory, fish were thawed, re-measured, and dissected. The recovery of trophically transmitted parasites from the body cavity, stomachs, intestines, and muscle followed standard necropsy procedures (Arthur \& Albert 1994). Recovered parasites were preserved in $70 \%$ ethanol. Most specimens were identified to species. To confirm parasite identification, parasites were compared to a reference collection (R. Olson pers. data) and to type specimens from the US National Parasite Collection, Beltsville, Maryland. Parasites recovered from salmon that were of freshwater origin were not included in the analyses. 


\section{Parasite analyses}

Parasite communities of each of the 10 fish species were described using parasite prevalence (\% of fish infected) and mean intensity (the number of individuals of a parasite species divided by the number of infected hosts) as defined by Bush et al. (1997). To identify patterns in the parasite community data for June and August 2002, non-metric multidimensional scaling (MDS) ordinations were constructed (Clarke \& Gorley 2006) based on the Bray-Curtis similarity coefficient (Bray \& Curtis 1957). Each fish was considered an individual sampling unit (parasite infracommunity level), and parasite abundance data were square-root transformed to reduce skewness, stabilize the variance, and increase the importance of less abundant species in the analyses (Clarke \& Warwick 2001). A dummy parasite was included in the parasite abundance dataset to include uninfected fish into ordination analyses. The data from each cruise were kept separate because of variability in ocean conditions, and differences in fish abundance, distributions, and parasite taxa.

One-way multivariate analysis of similarities (ANOSIM) permutation tests (Clarke \& Green 1988) were used to determine whether parasite communities were different among the fish species. A Global $R$ value (the strength of the effect) close to or equal to 1 suggests that all communities within a group are more similar to each other than to different groups, and an $\mathrm{R}$ approximating 0 indicates that similarities within and between groups are the same (Clarke \& Warwick 2001). To identify indicator species of parasite communities, we used the similarity percentages (SIMPER) procedure (Clarke 1993). Agglomerative clustering of all parasites of a fish species (component community) was conducted for comparison with previously published diet data on the same fish (Miller et al. 2010). All community analyses were performed using PRIMER (version 6.0; Clarke \& Warwick 2001). Other statistical analyses were run with Statview (SAS).

\section{Analysis for correlations between parasite abundances and environmental variables}

The correlations between parasite community structure and measured environmental variables for each cruise were examined using the BIO-ENV procedure (Clarke \& Ainsworth 1993). Rank correlations were calculated between a similarity matrix derived from parasite abundance data and matrices derived from various subsets of environmental variables (e.g. chlorophyll concentration, temperature, and salinity, recorded at $3 \mathrm{~m}$ depth at each station), thereby defining which environmental variables best explain the biotic structure. All BIO-ENV analyses were performed using PRIMER (version 6.0; Clarke \& Warwick 2001). All other statistical analyses were performed using the statistical program Statview (SAS).

\section{Hotspot analyses}

Several biological parameters were examined to identify biological activity occurring in the study area: chlorophyll concentrations, surface zooplankton biovolume and diversity, nekton abundance, nekton biomass, and nekton species diversity. Oceanographic biological hotspots were identified by Reese \& Brodeur (2006) (Fig. 1), who defined a biological hotspot in this region as an area with greater than average biological activity in terms of nekton species richness, density, or biomass.

The locations of these biological hotspots, off Hecata Bank, Oregon, and Crescent City, California, were found to persist across seasons and years, yet varied with respect to pelagic fish community composition (Reese \& Brodeur 2006). Reese \& Brodeur (2006) used geostatistical modeling techniques to create surfaces of nekton abundance, biomass, and species richness to identify the locations of the biological hotspots over seasons and years. The surfaces were then combined in a Geographic Information System (GIS) to identify areas meeting the criteria of a hotspot as defined above (Reese \& Brodeur 2006). Here, we utilized these same areas that were previously determined to be biological hotspots off the Oregon coast. Furthermore, the data used to determine the locations of the biological hotspots were collected over the same time period as this current study, which allowed us to plot values of several features related to fish and parasites obtained from multiple sampling stations throughout the study area in relation to these biological hotspots. Values that were plotted for each of the sampled stations (both inside and outside of biological hotspots) include the total number of fish included in parasite sampling, prevalence of all fish infected, mean parasite richness of all fish caught, and mean abundance of parasites (the number of individuals of a parasite species divided by the number of hosts examined) from all fish caught at a station. 
Table 1. Prevalence (\%) of trophically transmitted parasites recovered from pelagic fishes caught off Oregon and northern California, USA, in June 2002. Sample size of fish species in parentheses below common name. All fish except salmonids were adult.

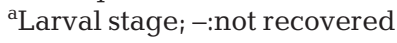

\begin{tabular}{|c|c|c|c|c|c|c|c|c|c|c|c|}
\hline Parasite taxon & $\begin{array}{c}\text { Chinook } \\
\text { sub- } \\
\text { adult } \\
\text { (53) }\end{array}$ & $\begin{array}{c}\text { Salmon } \\
\text { Chinook } \\
\text { year- } \\
\text { ling } \\
(73)\end{array}$ & $\begin{array}{l}\text { Coho } \\
\text { year- } \\
\text { ling } \\
(120)\end{array}$ & $\begin{array}{c}\text { Northern } \\
\text { anchovy } \\
(32)\end{array}$ & $\begin{array}{c}\text { Sn } \\
\text { Pacific } \\
\text { herring } \\
(50)\end{array}$ & $\begin{array}{c}\text { nall pela } \\
\text { Pacific } \\
\text { sar- } \\
\text { dine } \\
(63)\end{array}$ & $\begin{array}{c}\text { agics - } \\
\text { Pacific } \\
\text { saury } \\
(26)\end{array}$ & $\begin{array}{c}\text { Surf } \\
\text { smelt } \\
(46)\end{array}$ & $\begin{array}{c}\text { White- } \\
\text { bait } \\
\text { smelt } \\
(60)\end{array}$ & $\begin{array}{c}\text { Large } p \\
\text { Jack } \\
\text { mack- } \\
\text { erel } \\
(60)\end{array}$ & $\begin{array}{c}\text { oelagics } \\
\text { Pacific } \\
\text { hake } \\
(30)\end{array}$ \\
\hline Acanthocephala & & & & & & & & & & & \\
\hline Rhadinorhynchus sp. & 5.7 & 1.4 & - & - & - & - & 76.9 & 2.2 & 1.7 & 90.0 & - \\
\hline Cestoda & & & & & & & & & & & \\
\hline Bothriocephalus opsariichthydis & - & - & - & - & - & - & - & - & - & - & 46.7 \\
\hline Bothriocephalus sp. ${ }^{\mathrm{a}}$ & 17.0 & 12.3 & 3.3 & - & - & - & - & - & - & 1.7 & - \\
\hline Tetraphyllid sp. $1^{\mathrm{a}}$ & 17.0 & 4.1 & 1.7 & - & - & - & 7.7 & - & 1.7 & 30.0 & - \\
\hline Tetraphyllid sp. $2^{\mathrm{a}}$ & 5.7 & 1.4 & 1.7 & - & - & - & - & - & - & 5.0 & - \\
\hline Nematoda & & & & & & & & & & & \\
\hline Anisakis spp. ${ }^{\mathrm{a}}$ & 68.0 & 12.3 & 6.7 & 3.1 & 62.0 & 22.2 & & 10.9 & 3.3 & 100.0 & 70.0 \\
\hline Hysterothylacium aduncum & 85.0 & 28.8 & 15.0 & 1.2 & 12.0 & 9.5 & 3.8 & 4.3 & 6.7 & 40.0 & 16.7 \\
\hline Cucullanus sp. & - & 2.7 & 4.2 & - & - & - & - & - & - & - & - \\
\hline Unidentified nematode & - & - & - & - & 4.0 & - & - & - & - & - & - \\
\hline Trematoda & & & & & & & & & & & \\
\hline Brachyphallus crenatus & 3.8 & 1.4 & 1.7 & - & - & - & - & - & - & - & - \\
\hline Hemiurus levinseni & 62.3 & 50.7 & 16.7 & 3.1 & 6.0 & - & - & 10.9 & 20.0 & - & - \\
\hline Lecithaster gibbosus & 34.0 & 22.0 & 18.3 & - & - & - & - & 2.2 & - & 3.3 & - \\
\hline Lecithochirum sp. & - & - & - & - & - & - & - & - & - & 1.7 & - \\
\hline Myosaccium ecaude & - & - & - & - & - & 1.6 & - & - & - & - & - \\
\hline Parahemiurus merus & - & - & - & 6.2 & 8.0 & 14.3 & - & - & - & - & - \\
\hline Pronoprymna petrowi & - & - & - & - & - & - & - & 2.2 & 3.3 & - & - \\
\hline Aponurus sp. & - & - & - & - & - & - & - & - & - & - & 3.3 \\
\hline Derogenes varicus & - & - & - & - & - & - & - & - & - & - & 3.3 \\
\hline
\end{tabular}

Table 2. Prevalence (\%) of trophically transmitted parasites recovered from pelagic fishes caught off Oregon and northern California, USA, in August 2002. Otherwise as in Table 1 legend

\begin{tabular}{|c|c|c|c|c|c|c|c|c|c|c|c|}
\hline Parasite taxon & $\begin{array}{l}\text { Chinook } \\
\text { sub- } \\
\text { adult } \\
\text { (26) }\end{array}$ & $\begin{array}{l}\text { Salmon } \\
\text { Chinook } \\
\text { year- } \\
\text { ling } \\
(28)\end{array}$ & $\begin{array}{l}\text { Coho } \\
\text { year- } \\
\text { ling } \\
(31)\end{array}$ & $\begin{array}{l}\text { Northern } \\
\text { anchovy } \\
(49)\end{array}$ & $\begin{array}{l}\mathrm{Sr} \\
\text { Pacific } \\
\text { herring } \\
\quad(57)\end{array}$ & $\begin{array}{l}\text { nall pelc } \\
\text { Pacific } \\
\text { sar- } \\
\text { dine } \\
(59)\end{array}$ & $\begin{array}{l}\text { agics } \\
\text { Pacific } \\
\text { saury } \\
(122)\end{array}$ & $\begin{array}{l}\text { Surf } \\
\text { smelt } \\
(60)\end{array}$ & $\begin{array}{c}\text { White- } \\
\text { bait } \\
\text { smelt } \\
(41)\end{array}$ & \multicolumn{2}{|c|}{\begin{tabular}{cc}
\multicolumn{2}{c}{ Large pelagics } \\
Jack & Pacific \\
mack- & hake \\
erel & $(57)$ \\
$(60)$ &
\end{tabular}} \\
\hline Acanthocephala & & & & & & & & & & & \\
\hline Rhadinorhynchus sp. & 3.8 & - & 3.2 & - & - & - & 8.2 & - & - & 91.7 & - \\
\hline $\begin{array}{l}\text { Cestoda } \\
\text { Bothriocephalus opsariichthydis } \\
\text { Bothriocephalus sp. }{ }^{\mathrm{a}} \\
\text { Tetraphyllid sp. } \mathrm{1}^{\mathrm{a}}\end{array}$ & $\begin{array}{r}15.4 \\
34.6\end{array}$ & $\begin{array}{c}- \\
39.3 \\
10.7\end{array}$ & $\begin{array}{r}- \\
6.4 \\
12.9\end{array}$ & $\begin{array}{l}- \\
- \\
-\end{array}$ & $\begin{array}{l}- \\
- \\
-\end{array}$ & $\begin{array}{l}- \\
- \\
-\end{array}$ & $\begin{array}{l}- \\
- \\
-\end{array}$ & $\begin{array}{l}- \\
- \\
-\end{array}$ & $\begin{array}{l}- \\
- \\
-\end{array}$ & $\begin{array}{l}- \\
- \\
6.7\end{array}$ & $\begin{array}{c}82.5 \\
- \\
1.7\end{array}$ \\
\hline $\begin{array}{l}\text { Nematoda } \\
\text { Anisakis spp. }^{\text {a }} \\
\text { Hysterothylacium aduncum } \\
\text { Cucullanus sp. } \\
\text { Unidentified nematode }\end{array}$ & $\begin{array}{c}53.8 \\
19.2 \\
3.8 \\
-\end{array}$ & $\begin{array}{l}25 \\
10.7 \\
10.7 \\
-\end{array}$ & $\begin{array}{c}16.1 \\
45.2 \\
3.2 \\
-\end{array}$ & $\begin{array}{c}18.4 \\
6.1 \\
- \\
-\end{array}$ & $\begin{array}{l}57.9 \\
7 \\
- \\
-\end{array}$ & $\begin{array}{c}11.9 \\
6.8 \\
- \\
-\end{array}$ & $\begin{array}{l}- \\
- \\
- \\
-\end{array}$ & $\begin{array}{l}10 \\
3.3 \\
- \\
-\end{array}$ & $\begin{array}{l}4.9 \\
2.4 \\
- \\
-\end{array}$ & $\begin{array}{c}100 \\
11.7 \\
- \\
1.6\end{array}$ & $\begin{array}{c}71.9 \\
15.8 \\
- \\
-\end{array}$ \\
\hline $\begin{array}{l}\text { Trematoda } \\
\text { Brachyphallus crenatus } \\
\text { Hemiurus levinseni } \\
\text { Lecithaster gibbosus } \\
\text { Myosaccium ecaude } \\
\text { Parahemiurus merus } \\
\text { Tubulovesicula sp. } \\
\text { Pronoprymna petrowi }\end{array}$ & $\begin{array}{l}3.8 \\
53.8 \\
11.5 \\
- \\
- \\
- \\
-\end{array}$ & $\begin{array}{l}- \\
71.4 \\
21.4 \\
- \\
- \\
- \\
-\end{array}$ & $\begin{array}{r}9.7 \\
48.4 \\
22.6 \\
- \\
- \\
- \\
-\end{array}$ & $\begin{array}{l}- \\
- \\
- \\
- \\
30.6 \\
- \\
-\end{array}$ & $\begin{array}{l}- \\
5.3 \\
- \\
- \\
10.5 \\
- \\
-\end{array}$ & $\begin{array}{l}- \\
- \\
- \\
8.5 \\
15.2 \\
- \\
-\end{array}$ & $\begin{array}{l}- \\
- \\
- \\
- \\
- \\
5.7 \\
-\end{array}$ & $\begin{array}{l}- \\
- \\
- \\
- \\
- \\
- \\
1.7\end{array}$ & $\begin{array}{l}- \\
7.3 \\
- \\
- \\
- \\
- \\
-\end{array}$ & $\begin{array}{l}- \\
- \\
- \\
- \\
- \\
1.7 \\
-\end{array}$ & $\begin{array}{l}- \\
- \\
- \\
- \\
- \\
-\end{array}$ \\
\hline
\end{tabular}




\section{RESULTS}

Nineteen different parasite taxa representing 4 phyla were recovered from 10 species of fish caught during June (Table 1) and August 2002 (Table 2). The number of parasite species recovered ranged from 2 species infecting Pacific saury in August to 10 species infecting yearling Chinook salmon in June. The salmonids consistently had the greatest number of trophically transmitted parasite species (8 to 10). The only other fish species with parasite species richness similar to salmonids was jack mackerel, which had a maximum of 8 parasite species. We recovered the least number of parasites from Pacific saury in both June (3 species) and August (2 species).

The parasites recovered included both generalists (found in many unrelated fish species) and specialists (found in only 1 or a few related fish species). Hysterothylacium aduncum (Nematoda) was recovered from all fish species and larval Anisakis spp. (Nematoda) were recovered from all fish species except Pacific saury. Specialist parasite species included Myosaccium ecaude (Trematoda) that infects only Pacific sardine, and Bothriocephalus opsariichthydis
(Cestoda) recovered only from Pacific hake. Intensities of parasites were generally low, with means $<6.0$ worms per infected fish, except for larval Anisakis spp. in jack mackerel, which had an intensity (mean \pm SE) of $143.2 \pm 17.7$ in June (Table 3 ) and $97.4 \pm 11.3$ in August (Table 4).

\section{Host effects: parasite communities in June}

Parasite communities of individual fish caught in June were first compared through MDS ordinations (Fig. 2). There were significant differences in the parasite communities of the fish species examined (ANOSIM Global R of 0.372, p $\leq 0.001$; Fig. 2). Jack mackerel had the most distinctive parasite community compared to all other fish species (Fig. 2), with most pairwise comparisons resulting in ANOSIM $\mathrm{R}$ values $>0.9$. The parasite communities of Pacific saury were also significantly different from all other fish species, but pairwise comparison ANOSIM R values ranged from 0.292 (with yearling coho salmon) to 0.915 with jack mackerel. Pacific hake parasite communities differed from all other commu-

Table 3. Intensity (mean, SE in parentheses) of trophically transmitted parasites recovered from fish caught in June 2002. Sample size of fish examined in parentheses below their common name. ${ }^{*}$ parasites were recovered from 1 fish. ${ }^{a}$ Larval stage $;-:$ not recovered

\begin{tabular}{|c|c|c|c|c|c|c|c|c|c|c|c|}
\hline \multirow[t]{2}{*}{ Parasite taxon } & \multirow[b]{2}{*}{$\begin{array}{c}\text { Chinook } \\
\text { sub- } \\
\text { adult } \\
\text { (53) }\end{array}$} & \multirow[b]{2}{*}{$\begin{array}{c}\text { - Salmon - } \\
\text { Chinook } \\
\text { year- } \\
\text { ling } \\
\text { (73) }\end{array}$} & \multirow[b]{2}{*}{$\begin{array}{l}\text { Coho } \\
\text { year- } \\
\text { ling } \\
(120)\end{array}$} & \multirow[b]{2}{*}{$\begin{array}{c}\text { Northern } \\
\text { anchovy } \\
(32)\end{array}$} & \multicolumn{3}{|c|}{ - Small pelagics - } & \multirow[b]{2}{*}{$\begin{array}{c}\text { Surf } \\
\text { smelt } \\
(46)\end{array}$} & \multirow[b]{2}{*}{$\begin{array}{l}\text { White- } \\
\text { bait } \\
\text { smelt } \\
(60)\end{array}$} & \multicolumn{2}{|c|}{ Large pelagics } \\
\hline & & & & & $\begin{array}{l}\text { Pacific } \\
\text { herring } \\
\quad(50)\end{array}$ & $\begin{array}{c}\text { Pacific } \\
\text { sardine } \\
(63)\end{array}$ & $\begin{array}{c}\text { Pacific } \\
\text { saury } \\
(26)\end{array}$ & & & $\begin{array}{c}\text { Jack } \\
\text { mackerel } \\
(60)\end{array}$ & $\begin{array}{c}\text { Pacific } \\
\text { hake } \\
(30)\end{array}$ \\
\hline \multicolumn{12}{|l|}{ Acanthocephala } \\
\hline Rhadinorhynchus sp. & $1(0)$ & $2^{*}$ & - & - & - & - & $5.5(1.6)$ & 5) $1^{*}$ & $1^{*}$ & $5.3(0.6)$ & - \\
\hline Bothriocephalus sp. ${ }^{\mathrm{a}}$ & $1.4(0.2)$ & $1.6(0.3)$ & $1(0)$ & - & - & - & - & - & - & $2^{*}$ & - \\
\hline Tetraphyllid sp. $1^{\mathrm{a}}$ & $1.9(0.6)$ & $1.3(0.3)$ & $1(0)$ & - & - & - & $1(0)$ & - & $1^{*}$ & $2.3(0.5)$ & - \\
\hline Tetraphyllid sp. $2^{\mathrm{a}}$ & $1(0)$ & $4^{*}$ & $1.5(0.5)$ & - & - & - & - & - & - & $1.3(0.3)$ & - \\
\hline \multicolumn{12}{|l|}{ Nematoda } \\
\hline Anisakis spp. ${ }^{\mathrm{a}}$ & $2.8(0.4)$ & $1.6(0.4)$ & $1(0)$ & $1^{*}$ & $4.0(0.7)$ & $3.1(1.1)$ & - & $1(0)$ & $1(0) 1$ & $143.2(17.7)$ & $3.3(0.7)$ \\
\hline Hysterothylacium aduncum & $2.9(0.3)$ & $1.6(0.3)$ & $1.9(0.4)$ & $1^{*}$ & $1(0)$ & $1.5(0.3)$ & $1(0)$ & $1.5(0.5)$ & $1(0)$ & $1.6(0.2)$ & $1(0)$ \\
\hline Hemiurus levinseni & $5.5(1.1)$ & $4.4(0.8)$ & $1.4(0.1)$ & $2^{*}$ & $3.7(2.2)$ & - & - & $1.2(0.2)$ & $7.2(2.6)$ & - & - \\
\hline Lecithaster gibbosus & $3.4(2.7)$ & $2.9(0.7)$ & $2.4(0.5)$ & - & - & - & - & $1^{*}$ & - & $1(0)$ & - \\
\hline Lecithochirum sp. & - & - & - & - & - & - & - & - & - & $4.0^{*}$ & - \\
\hline Myosaccium ecaude & - & - & - & - & - & $1^{*}$ & - & - & - & - & - \\
\hline Parahemiurus merus & - & - & - & $1.5(0.5)$ & $1.2(0.2)$ & $1.4(0.4)$ & - & - & - & - & - \\
\hline Pronoprymna petrowi & - & - & - & - & - & - & - & $2^{*}$ & $1(0)$ & - & - \\
\hline Aponurus sp. & - & - & - & - & - & - & - & - & - & - & $1^{*}$ \\
\hline Derogenes varicus & - & - & - & - & - & - & - & - & - & - & $1^{*}$ \\
\hline
\end{tabular}


Table 4. Intensity (mean, SE in parentheses) of trophically transmitted parasites recovered from fish caught in August 2002. Sample size of fishes examined in parentheses below common names. Otherwise as in Table 3 legend

\begin{tabular}{|c|c|c|c|c|c|c|c|c|c|c|c|}
\hline \multirow{2}{*}{ Parasite taxon } & \multirow[b]{2}{*}{$\begin{array}{c}\text { Chinook } \\
\text { sub- } \\
\text { adult } \\
(26)\end{array}$} & \multirow[b]{2}{*}{$\begin{array}{c}\text { Salmon } \\
\text { Chinook } \\
\text { year- } \\
\text { ling } \\
(28)\end{array}$} & \multirow[b]{2}{*}{$\begin{array}{l}\text { Coho } \\
\text { year- } \\
\text { ling } \\
(31)\end{array}$} & \multirow[b]{2}{*}{$\begin{array}{c}\text { Northern } \\
\text { anchovy } \\
\text { (49) }\end{array}$} & \multirow{2}{*}{$\begin{array}{c}\mathrm{Sn} \\
\text { Pacific } \\
\text { herring } \\
\quad(57)\end{array}$} & \multirow[b]{2}{*}{$\begin{array}{l}\text { Aall pelag } \\
\text { Pacific } \\
\text { sardine } \\
\text { (59) }\end{array}$} & \multirow{2}{*}{$\begin{array}{l}\text { Pics } \\
\text { Pacific } \\
\text { saury } \\
(122)\end{array}$} & \multirow[b]{2}{*}{$\begin{array}{c}\text { Surf } \\
\text { smelt } \\
(60)\end{array}$} & \multirow[b]{2}{*}{$\begin{array}{c}\text { White- } \\
\text { bait } \\
\text { smelt } \\
(41)\end{array}$} & \multicolumn{2}{|c|}{ Large pelagics } \\
\hline & & & & & & & & & & $\begin{array}{l}\text { Jack } \\
\text { mackerel } \\
(60)\end{array}$ & $\begin{array}{c}\text { Pacific } \\
\text { hake } \\
(57)\end{array}$ \\
\hline \multicolumn{12}{|l|}{ Acanthocephala } \\
\hline Rhadinorhynchus sp. & $1.0^{*}$ & - & $1.0^{*}$ & - & - & - & $1.1(0.1)$ & - & - & $6.4(0.7)$ & - \\
\hline Bothriocephalus sp. ${ }^{\mathrm{a}}$ & $1.2(0.2)$ & $1.4(0.1)$ & $1.0(0)$ & - & - & - & - & - & - & - & - \\
\hline Tetraphyllid sp. $1^{\mathrm{a}}$ & $2.1(0.3)$ & $1.3(0.3)$ & $1.0(0)$ & - & - & - & - & - & - & $1.7(0.5)$ & $1.0(0)$ \\
\hline \multicolumn{12}{|l|}{ Nematoda } \\
\hline Anisakis spp. ${ }^{\mathrm{a}}$ & $2.0(0.3)$ & $1.3(0.2)$ & $1.2(0.2)$ & $1.7(0.3)$ & $3.2(0.4)$ & $3.1(1.8)$ & - & $1.0(0)$ & $1.5(0.5)$ & $97.4(11.3)$ & $2.5(0.3)$ \\
\hline Hysterothylacium aduncum & $1.8(0.4)$ & $1.3(0.3)$ & $2.6(0.6)$ & $1.0(0)$ & $1.2(0.2)$ & $4.5(2.6)$ & - & $1.0(0)$ & $1.0^{*}$ & $1.1(0.1)$ & $1.0(0)$ \\
\hline Cucullanus sp. & $2.0^{*}$ & $2.3(0.7)$ & $1.0^{*}$ & - & - & - & - & - & - & - & - \\
\hline Myosaccium ecaude & - & - & - & - & - & $4.6(2.4)$ & - & - & - & - & - \\
\hline Parahemiurus merus & - & - & - & $1.6(0.2)$ & $1.3(0.3)$ & $2.6(1.1)$ & - & - & - & - & - \\
\hline Tubulovesicula sp. & - & - & - & - & - & - & $1.0(0)$ & - & - & $1.0^{*}$ & - \\
\hline Pronoprymna petrowi & - & - & - & - & - & - & - & $2.0^{*}$ & - & - & - \\
\hline
\end{tabular}

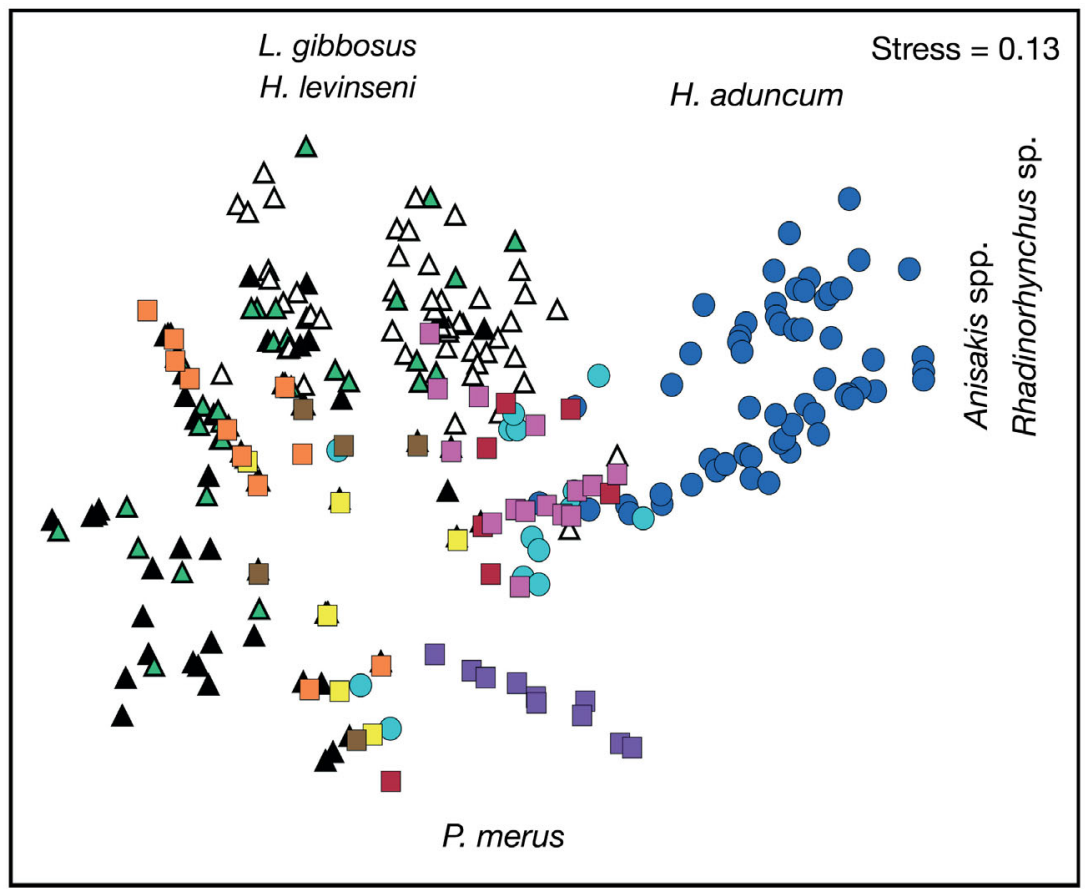

$\triangle$ Chinook Salmon subadult $\Delta$ Chinook Salmon yearling $\triangle$ Coho Salmon yearling $\square$ Northern Anchovy $\square$ Pacific Herring Pacific Sardine $\square$ Pacific Saury Surf Smelt $\square$ Whitebait Smelt - Jack Mackerel Pacific Hake

Fig. 2. Non-metric multi-dimensional scaling plots using square-root transformed parasite abundance data from 10 fish species collected during June 2002. Each symbol represents an individual fish. Symbol shapes: salmon $=\Delta$, small pelagics $=\square$, or large pelagics $=0$. Chinook salmon are separated into yearlings and subadults. Coho salmon were yearlings, and the remaining fish species were all adults. Parasites that best describe the ordinations are: Anisakis spp., Hemiurus levinseni, Hysterothylacium aduncum, Lecithaster gibbosus, Parahemiurus merus, and Rhadinorhynchus sp. These genera are indicated in their general direction of greatest abundance 
nities; however, the strength of the difference with those of Pacific herring was very small (ANOSIM R = 0.105, $\mathrm{p}=0.002$ ).

Using a SIMPER analysis, specific parasites were identified as indicator species responsible for the patterns described by the MDS ordination. Those parasite species contributing $>10 \%$ to the community are listed in Table 5. The nematodes Anisakis spp. were the indicator species of jack mackerel parasite communities, although overlaps among communities observed in the MDS plot (Fig. 2) were in part due to Anisakis spp. being recovered in other fish species such as Pacific hake and Pacific herring (Table 5). The abundance of the acanthocephalan Rhadinorhynchus sp. in the parasite communities of both Pacific saury and jack mackerel also resulted in some overlap in the MDS. The indicator species of parasite communities of yearling coho salmon were the trematodes Hemiurus levenseni and Lecithaster gibbosus. H. levenseni was also the indicator species of yearling Chinook salmon parasite communities. For subadult Chinook salmon, the nematode Hysterothylacium aduncum was the indicator species.

Cluster dendrograms of percent similarity between parasite communities of fish species (abundances aver- aged by all fish of a species) show simplified and similar relationships to those shown by the MDS plots and the ANOSIM results generated from individual fish (Fig. 3). Pacific hake and Pacific herring had parasite communities most similar to each other, followed by high similarity between the yearling salmonids and between the smelts. As also seen with individual fish, jack mackerel and Pacific saury parasite communities had the least similarity with all other fish species.

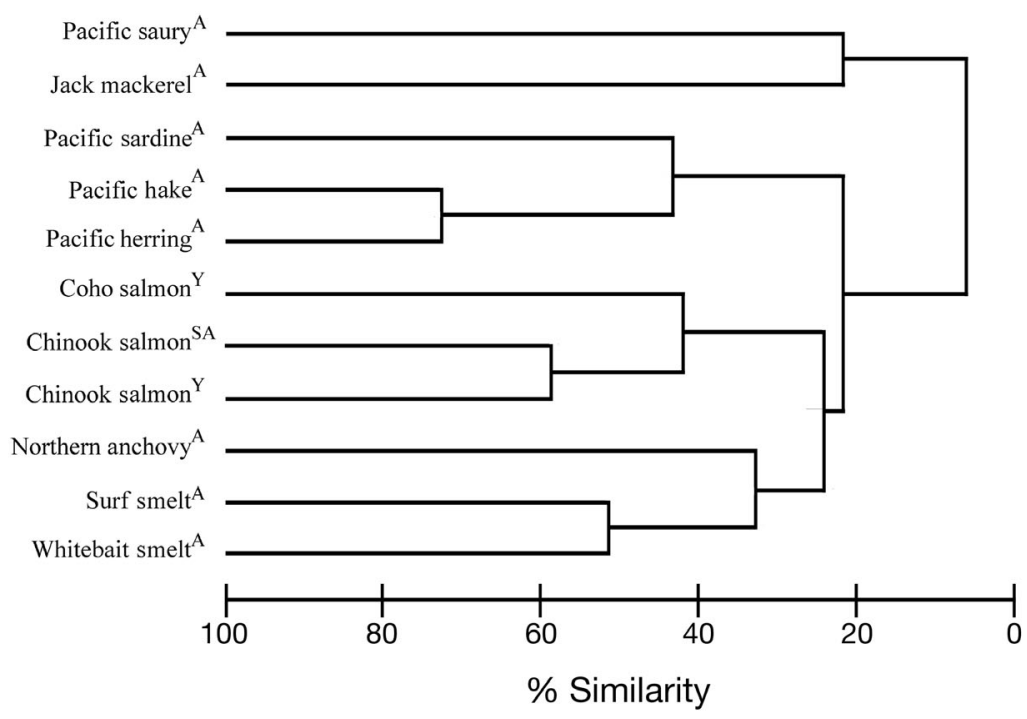

Fig. 3. Cluster dendrogram using square-root transformed parasite abundance data that were then averaged by fish species collected during June 2002. Ages classes of fishes: $\mathrm{A}=$ adult, $\mathrm{SA}=$ subadult, and $\mathrm{Y}=$ yearling

Table 5. Percent contribution of indicator species (based upon abundance) that contributed $<10 \%$ to parasite communities of pelagic fish caught in June 2002. Sample size per fish species in parentheses below their common name. ${ }^{a}$ Larval stage; $-:$ no data

\begin{tabular}{|c|c|c|c|c|c|c|c|c|c|c|c|}
\hline \multirow[t]{2}{*}{ Parasite taxon } & \multicolumn{3}{|c|}{ Salmon } & \multirow[b]{2}{*}{$\begin{array}{c}\text { Northern } \\
\text { anchovy } \\
(32)\end{array}$} & \multirow{2}{*}{$\begin{array}{c}\text { Sn } \\
\text { Pacific } \\
\text { herring } \\
(50)\end{array}$} & \multicolumn{2}{|c|}{ nall pelagics } & \multirow[b]{2}{*}{$\begin{array}{c}\text { Surf } \\
\text { smelt } \\
(46)\end{array}$} & \multirow[b]{2}{*}{$\begin{array}{c}\text { White- } \\
\text { bait } \\
\text { smelt } \\
(60)\end{array}$} & \multicolumn{2}{|c|}{ Large pelagics } \\
\hline & $\begin{array}{c}\text { Chinook } \\
\text { sub- } \\
\text { adult } \\
\text { (53) }\end{array}$ & $\begin{array}{c}\text { Chinook } \\
\text { year- } \\
\text { ling } \\
(73)\end{array}$ & $\begin{array}{l}\text { Coho } \\
\text { year- } \\
\text { ling } \\
(120)\end{array}$ & & & $\begin{array}{l}\text { Pacific } \\
\text { sar- } \\
\text { dine } \\
(63)\end{array}$ & $\begin{array}{c}\text { Pacific } \\
\text { saury } \\
(26)\end{array}$ & & & $\begin{array}{c}\text { Jack } \\
\text { mack- } \\
\text { erel } \\
(60)\end{array}$ & $\begin{array}{c}\text { Pacific } \\
\text { hake } \\
(30)\end{array}$ \\
\hline \multicolumn{12}{|l|}{ Acanthocephala } \\
\hline Rhadinorhynchus sp. & - & - & - & - & - & - & 99.7 & - & - & 16.4 & - \\
\hline \multicolumn{12}{|l|}{ Cestoda } \\
\hline Bothriocephalus opsariichthydis & - & - & - & - & - & - & - & - & - & - & 26.2 \\
\hline \multicolumn{12}{|l|}{ Nematoda } \\
\hline Anisakis spp. ${ }^{\mathrm{a}}$ & 25.7 & - & - & - & 96.2 & 63.4 & - & 55.9 & - & 80.9 & 72.0 \\
\hline Hysterothylacium aduncum & 45.6 & 15.1 & 25.2 & - & - & - & - & - & - & - & - \\
\hline \multicolumn{12}{|l|}{ Trematoda } \\
\hline Hemiurus levinseni & 20.9 & 69.4 & 34.5 & - & - & - & - & 39.8 & 85.9 & - & - \\
\hline Lecithaster gibbosus & - & 10.8 & 32.8 & - & - & - & - & - & - & - & - \\
\hline Myosaccium ecaude & - & - & - & - & - & - & - & - & - & - & - \\
\hline Parahemiurus merus & - & - & - & 100.0 & - & 29.1 & - & - & - & - & - \\
\hline
\end{tabular}




\section{Host effects: parasite communities in August}

Although the general patterns of trophic relationships were similar to those observed in June, we found greater differences among the parasite communities of individual fish species in August than June (ANOSIM Global $\mathrm{R}=0.508 \mathrm{p} \leq 0.001$; Fig. 4). Jack mackerel parasite communities again differed from all other parasite communities (Fig. 4), with ANOSIM R values ranging from 0.873 (a pairwise comparison with Pacific herring, $\mathrm{p} \leq 0.001$ ) to 0.998 (a pairwise comparison with Pacific saury, $\mathrm{p} \leq 0.001$ ). Parasite communities of Pacific hake caught in August also differed from communities of most other fish species (Fig. 4). Overlaps between parasite communities were greatest among some of the forage fish species (among the 2 smelt species, anchovy and sardine). Parasite communities of several other forage fish species, including Pacific sardine and Pacific herring, were split into 2 groups in the MDS: those with and without the trematode Parahemiurus merus (Fig. 4). The parasite communities of salmonids were also split into 2 groups in the MDS (Fig. 4) and differed from all parasite communities of nonsalmonids.
Indicator species analysis identified Anisakis spp. as the largest contributors to the parasite communities of many of the pelagic fish examined, especially Pacific herring and surf smelt (Table 6). For jack mackerel, parasite communities were defined by Anisakis spp. and Rhadinorhynchus sp. The parasite communities of Pacific saury were also defined by Rhadinorhynchus sp., and communities of Pacific hake were defined by Bothriocephalus opsariichthydis and Anisakis spp. The trematode Hemiurus levinseni was the indicator species for yearling coho salmon, Chinook salmon, and whitebait smelt parasite communities. Both northern anchovy and Pacific sardine had the trematode Parahemiurus merus as the largest contributor to their parasite communities.

A cluster dendrogram of percent similarity among parasite communities of fish species (abundances averaged by all fish of a species) caught in August (Fig. 5) shows simplified and similar relationships as ANOSIM results on individual fish parasite communities. In August, parasite communities of northern anchovy and Pacific sardine had high similarity, as well as the salmonids, the smelts, and again Pacific hake and Pacific herring. Jack mackerel and Pacific saury parasite communities again had the least similarity with all other fish species.

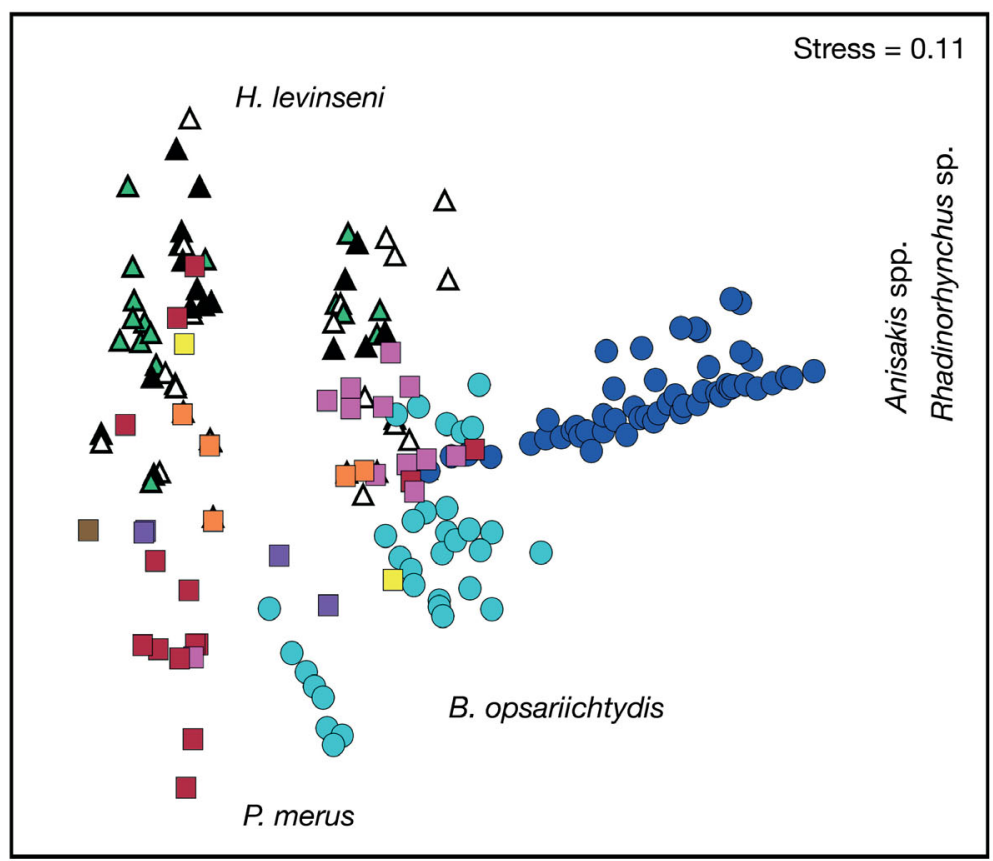

$\triangle$ Chinook Salmon subadult

$\Delta$ Chinook Salmon yearling

$\triangle$ Coho Salmon yearling

$\square$ Northern Anchovy

$\square$ Pacific Herring

- Pacific Sardine

$\square$ Pacific Saury

$\square$ Surf Smelt

$\square$ Whitebait Smelt

- Jack Mackerel

- Pacific Hake

Fig. 4. Non-metric multi-dimensional scaling plots using square-root transformed parasite abundance data from 10 fish species collected during August 2002. Each symbol represents an individual fish. Symbol shapes: salmon $=\Delta$, small pelagics $=\square$, or large pelagics $=0$. Chinook salmon are separated into yearlings and subadults. Coho salmon were yearlings and the remaining fish species were all adults. Parasites that best describe the ordinations are: Anisakis spp., Bothriocephalus opsariichthydis, Hemiurus levinseni, Parahemiurus merus, and Rhadinorhynchus sp. These genera are indicated in their general location of greatest abundance 
Table 6. Percent contribution of indicator species (based on abundance) that contributed $<10 \%$ to parasite communities of pel-

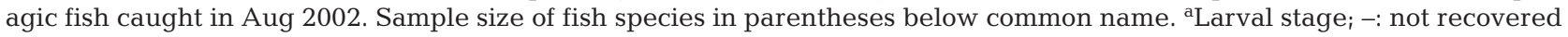

\begin{tabular}{|c|c|c|c|c|c|c|c|c|c|c|c|}
\hline Parasite taxon & $\begin{array}{l}\text { Chinook } \\
\text { sub- } \\
\text { adult } \\
\text { (26) }\end{array}$ & $\begin{array}{l}\text { Salmon - } \\
\text { Chinook } \\
\text { year- } \\
\text { ling } \\
(28)\end{array}$ & $\begin{array}{l}\text { Coho } \\
\text { year- } \\
\text { ling } \\
(31)\end{array}$ & $\begin{array}{c}\text { Northern } \\
\text { anchovy } \\
\text { (49) }\end{array}$ & $\begin{array}{c}\mathrm{Sn} \\
\text { Pacific } \\
\text { herring } \\
(57)\end{array}$ & $\begin{array}{c}\text { mall pela } \\
\text { Pacific } \\
\text { sar- } \\
\text { dine } \\
\text { (59) }\end{array}$ & $\begin{array}{l}\text { Pacifics } \\
\text { saury } \\
(122)\end{array}$ & $\begin{array}{c}\text { Surf } \\
\text { smelt } \\
(60)\end{array}$ & $\begin{array}{c}\text { White- } \\
\text { bait } \\
\text { smelt } \\
(41)\end{array}$ & \multicolumn{2}{|c|}{\begin{tabular}{cc}
\multicolumn{3}{c}{ Large pelagics } \\
Jack & Pacific \\
mack- & hake \\
erel & $(57)$ \\
$(60)$ &
\end{tabular}} \\
\hline \multicolumn{12}{|l|}{ Acanthocephala } \\
\hline Rhadinorhynchus sp. & - & - & - & - & - & - & 67.4 & - & - & 20.3 & - \\
\hline \multicolumn{12}{|l|}{ Cestoda } \\
\hline Bothriocephalus opsariichthydis & - & - & - & - & - & - & - & - & - & - & 61.5 \\
\hline Bothriocephalus sp. ${ }^{\mathrm{a}}$ & - & 17.1 & - & - & - & - & - & - & - & - & - \\
\hline Tetraphyllid sp. $1^{\mathrm{a}}$ & 15.0 & - & - & - & - & - & - & - & - & - & - \\
\hline \multicolumn{12}{|l|}{ Nematoda } \\
\hline Anisakis spp. ${ }^{\mathrm{a}}$ & 40.8 & - & - & 21.8 & 95.9 & 34.5 & - & 93.8 & 21.6 & 79.6 & 37.2 \\
\hline Hysterothylacium aduncum & - & - & 38.5 & - & - & - & - & - & - & - & - \\
\hline \multicolumn{12}{|l|}{ Trematoda } \\
\hline Hemiurus levinseni & 39.0 & 72.2 & 47.3 & - & - & - & - & - & 78.4 & - & - \\
\hline Myosaccium ecaude & - & - & - & - & - & 12.8 & - & - & - & - & - \\
\hline Parahemiurus merus & - & - & - & 77.0 & - & 45.8 & - & - & - & - & - \\
\hline Tubulovesicula sp. & - & - & - & - & - & - & 32.6 & - & - & - & - \\
\hline
\end{tabular}

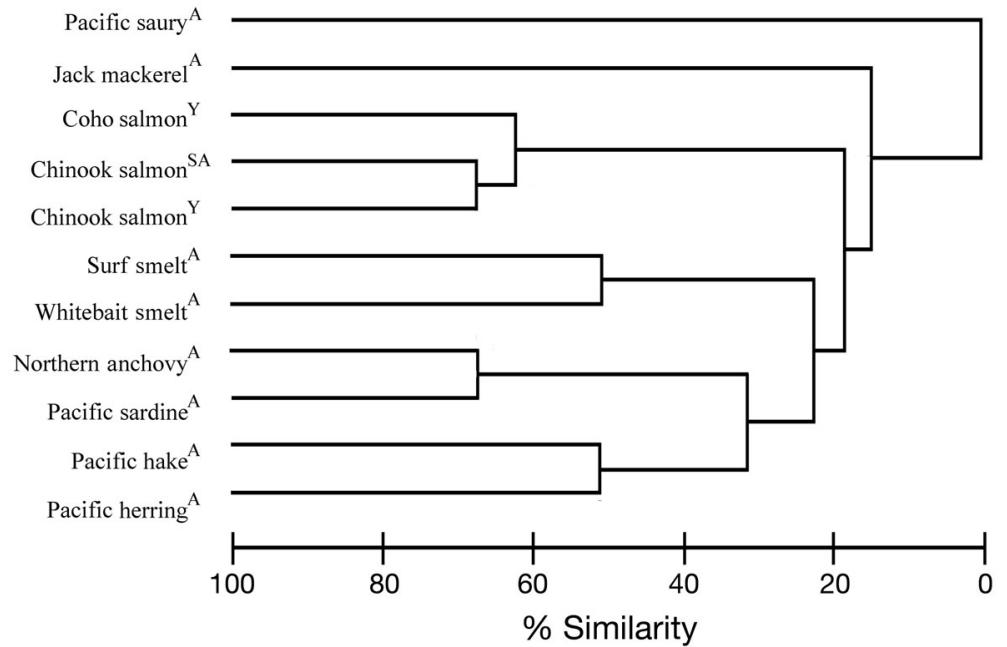

Fig. 5. Cluster dendrogram using square-root transformed parasite abundance data that were then averaged by fish species collected during August 2002. Age classes of fish: $\mathrm{A}=$ adult, $\mathrm{SA}=$ subadult, and $\mathrm{Y}=$ yearling

\section{Spatial and environmental effects}

We tested the hypothesis that physical oceanographic features associated with Cape Blanco act as physical barriers resulting in different marine communities to the north versus south of the cape and are reflected in the communities of trophically transmitted parasite communities of pelagic fishes. Nonmetric MDS analysis of parasite communities of all fish showed no effect of being caught north versus south of Cape Blanco in either June or August (ANOSIM Global $\mathrm{R}=-0.131$ and $-0.178, \mathrm{p}>0.05$, respectively). All fish species were included in this analysis regardless of whether they were caught in both locations. Those fish species that were caught both north and south of Cape Blanco in June were Pacific sardine, surf smelt, and whitebait smelt. In August, fish caught in both areas were Pacific saury and Pacific herring. Additional separate ANOSIMs run on only these species also reported no differences in the parasite communities north versus south of Cape Blanco (data not shown).

In addition, we tested for differences in parasite communities between fish caught at offshore versus inshore stations. In June, parasite communities were different between offshore and inshore stations (ANOSIM Global $\mathrm{R}=0.251, \mathrm{p} \leq 0.001$ ). Fish species caught both inshore and offshore included jack mackerel and Pacific sardines. In August, jack mackerel and Pacific saury were caught both inshore and offshore, but there was no cross-shelf difference in parasite communities.

We examined a suite of spatial and environmental variables for potential correlations with parasite communities, including station depth, distance offshore, latitude, as well as temperature, salinity, and 
chlorophyll. Spearman rank correlations (BEST BioEnv analysis) of June data indicated a weak positive correlation between both station depth (Rho = 0.242 ) and distance offshore (Rho $=0.222$ ) and parasite abundance. This was attributed largely to the abundance of the acanthocephalan Rhadinorhynchus sp. found primarily in fish caught offshore, such as Pacific saury and jack mackerel. We recovered approximately twice as many of these acanthocephalans from infected jack mackerel caught offshore (mean \pm SE intensity $6.4 \pm 1.1, \mathrm{n}=22$ ) than from jack mackerel caught in the nearshore (3.8 \pm $0.6, \mathrm{n}=38$ ) in June. The mean intensity of Rhadinorhynchus sp. in Pacific saury caught offshore ( $\mathrm{n}=$ 17) was similar $(6.1 \pm 1.9)$ to jack mackerel caught offshore. We only caught 5 Pacific saury inshore with a mean intensity of Rhadinorhynchus sp. of only $1.4( \pm 0.4)$. The intensity of Anisakis spp. also differed in infected jack mackerel caught at offshore and inshore stations in June, but those caught at offshore stations had significantly less $(p<0.05)$ than those at inshore stations $(72.6 \pm 20.1$ and $184.0 \pm$ 23.1, respectively). Temperature and salinity recorded at $3 \mathrm{~m}$ depth ranged from 7.8 to $13.3^{\circ} \mathrm{C}$ and from 30.10 to 34.0, respectively. Neither of these physical factors, nor chlorophyll concentration, had a significant effect on the trophically transmitted parasite communities (data not shown).

There was a weak positive correlation (Spearman rank correlation Rho $=0.158$ ) between chlorophyll and parasite abundance in August. In contrast to June, there were no significant differences in the intensities of either Rhadinorhynchus sp. or Anisakis spp., or any other parasite species, in jack mackerel caught at offshore and inshore stations. In August, the mean intensity of Rhadinorhynchus sp. was approximately 6.0, and the mean intensity of Anisakis spp. was approximately 100.0, in jack mackerel caught either inshore or offshore. The sea surface temperatures recorded at stations in August ranged from 8.3 to $13.8^{\circ} \mathrm{C}$, and salinity ranged from 32.20 to 33.95. Yet again, neither of these physical factors had a significant effect on any trophically transmitted parasite communities (data not shown).

\section{Hotspot analyses}

To test the hypothesis that exploitation of marine hotspots by fish would be reflected by a difference in the composition of trophically transmitted parasites, analyses were also conducted to determine whether parasites differed in fish caught inside versus outside
2 defined and persistent biological hotspots identified off of Heceta Head, Oregon, and Crescent City, California (Figs. $6 \&$ 7). We found that the parasite communities were not well defined by these hotspot locations and observed no differences in the parasite communities of any of the fish caught within either hotspot and those caught outside of a hotspot in June (ANOSIM Global $\mathrm{R}=0.054, \mathrm{p} \leq 0.001$ ) or August (ANOSIM Global R = -0.097, p > 0.05; MDS plots not shown).

In addition to comparisons of parasite community composition inside and outside of hotspots, we examined the overall prevalence of infection (the percentage of infected fish, with even a single parasite, versus uninfected), the total number of parasites (irrespective of parasite species), and species richness (the number of parasite species recovered). Generally, more fish were caught within hotspots $(57.1 \%$ of all fish in June and $73.2 \%$ in August); thus comparisons for individual fish species were limited to the species with sufficient sample sizes caught both within and outside of at least 1 hotspot (e.g. whitebait smelt, Pacific herring, Pacific sardine, northern anchovy, Chinook salmon, and coho salmon). We found no differences between overall prevalence, the total number of parasites, or species richness for individual fish species caught inside versus outside of hotspots in June or August (data not shown).

With the potential to measure differences in parasite communities inside versus outside of hotspots limited by sample sizes of some fish species, we also plotted the following for each haul of fish caught at a station (regardless of fish species composition): the total number of fish included in parasite sampling (Fig. 6A, June; Fig. 7A, August); the percent of all fish infected (Figs. 6B \& 7B); mean parasite richness of all fish caught at a station (Figs. 6C \& 7C); and mean number of parasites from all fish caught at a station (Figs. 6D \& 7D). There were no differences in either month for the prevalence, total number of parasites recovered from a haul of fish, or for parasite species richness within any fish species caught within or outside of a hotspot.

\section{DISCUSSION}

\section{Trophic structure of the pelagic food web}

The diversity of parasitic taxa recovered from the 10 pelagic fish species examined in this study reflected the trophic ecology of the pelagic ecosystem off of southern Oregon and northern California. 

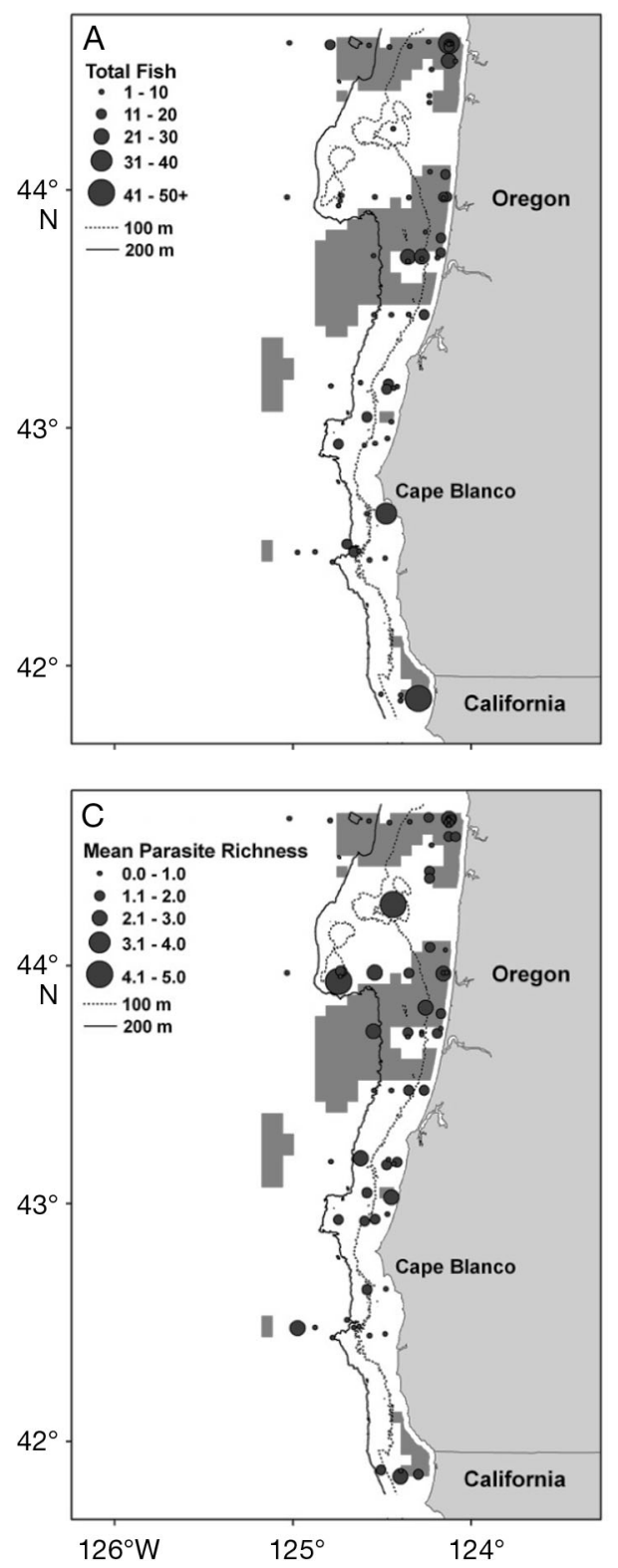
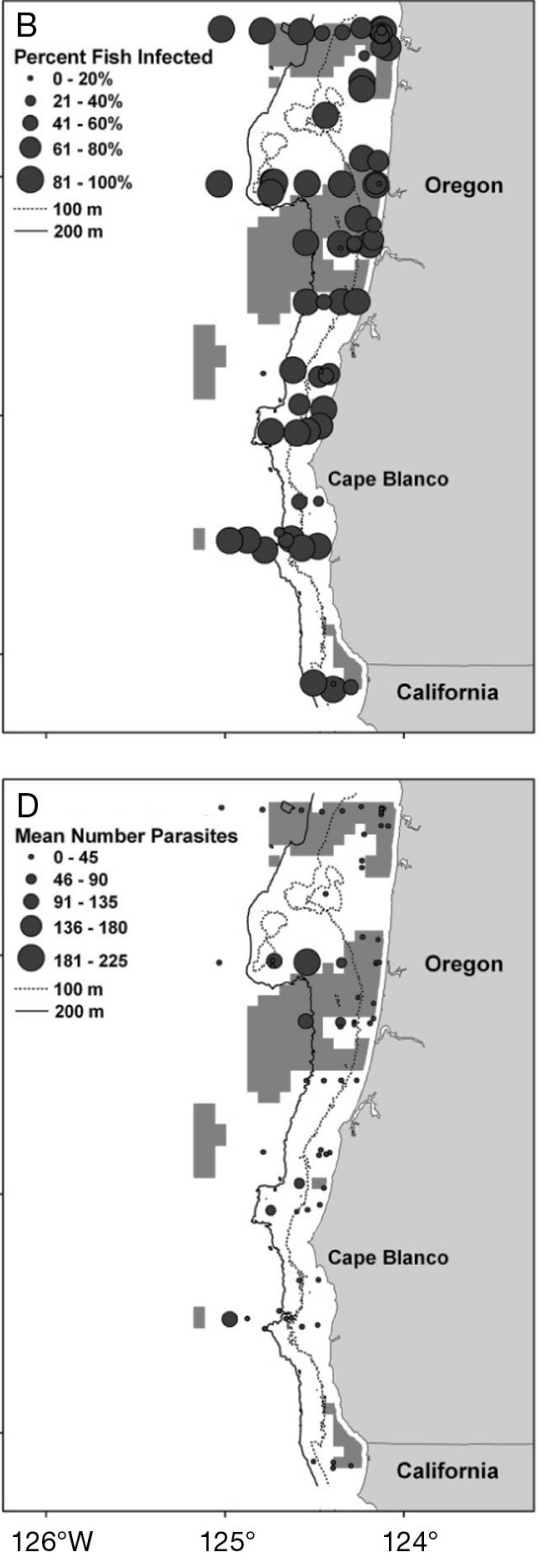

Fig. 6. Distributions of fish-parasite interactions observed in June 2002 relative to the locations of the nekton biological hotspots (shaded). (A) Total number of fish sampled, (B) percent of fish infected at each sample station, (C) mean parasite richness, and (D) mean number of parasites
Pacific herring, northern anchovy, Pacific sardine, Pacific saury, surf smelt, and whitebait smelt had parasite communities characterized by low species richness and low abundance, typical of fish with a low trophic position in a food web (Thompson et al. 2005, Valtonen et al. 2010). These 'forage fish' feed on phytoplankton and small zooplankton (van der Lingen et al. 2009), which are not prey preferred by juvenile salmon or adult predatory fish (Peterson et al. 1982, Brodeur \& Pearcy 1992, Miller \& Brodeur 2007). The more diverse parasite communities of juvenile salmon reflect their opportunistic feeding on invertebrates and vertebrates (Peterson et al. 1982,
Brodeur \& Pearcy 1992, Schabetsberger et al. 2003, Baldwin et al. 2008). Jack mackerel and Pacific hake had high prevalence and intensities of parasites typical of larger predatory fish that can feed higher up in the food web. These higher trophic level fish accumulate parasites both through greater feeding rates and consuming prey that have previously accumulated large quantities of parasites (Marcogliese 2005). For example, jack mackerel and Pacific hake had higher prevalence and intensities of larval Anisakis spp. that can be transferred from fish to fish through trophic interactions and also accumulate in an individual fish (Smith 1974). 

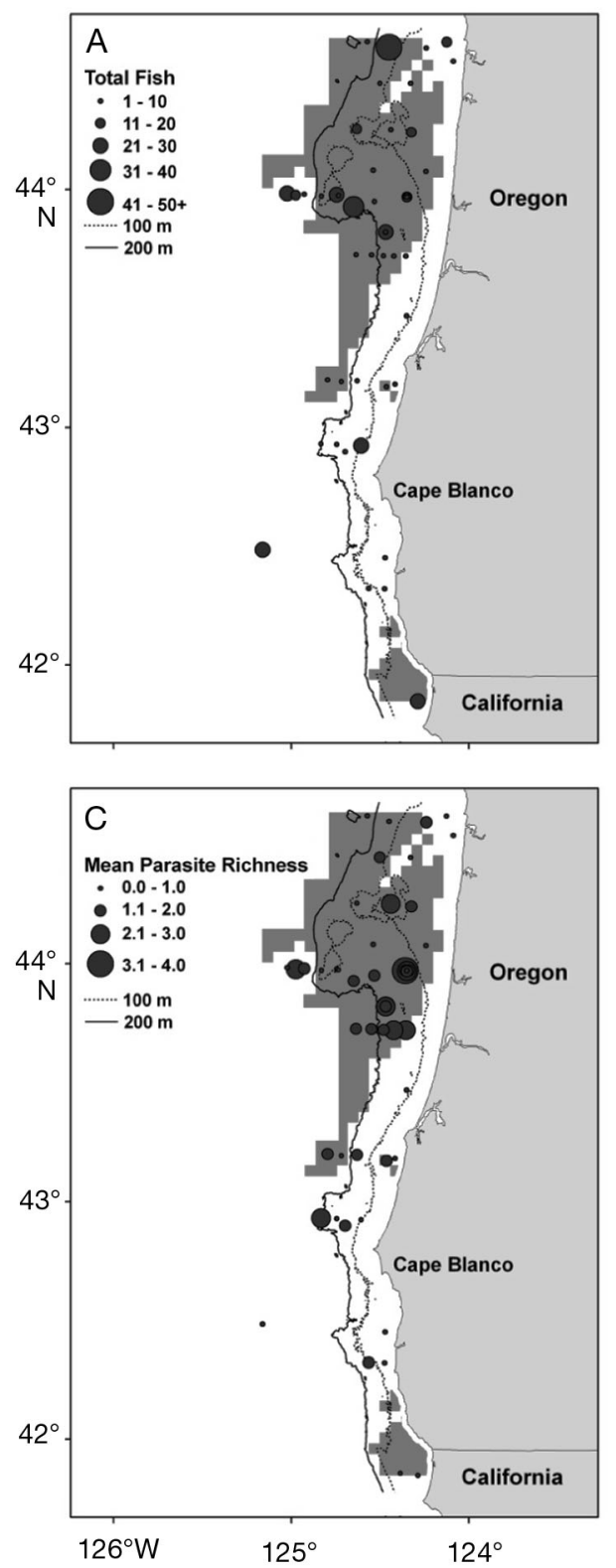
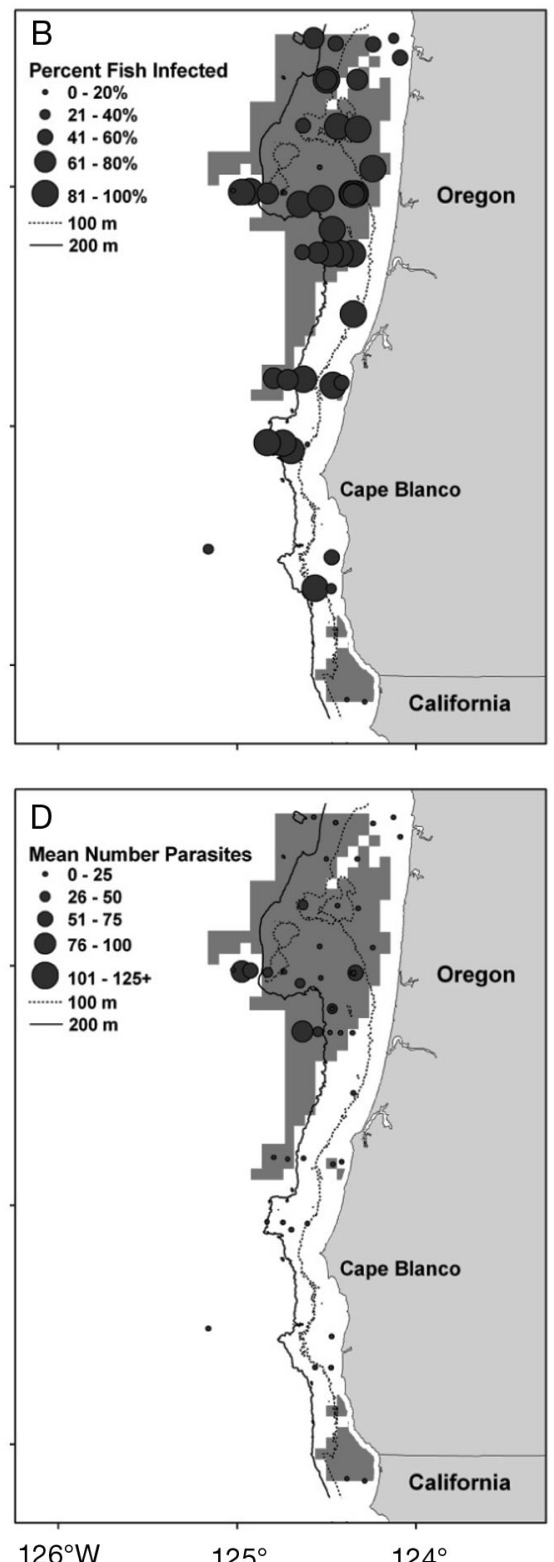

Fig. 7. Distributions of fish-parasite interactions observed in August 2002 relative to the locations of the nekton biological hotspots (shaded). (A) Total number of fish sampled, (B) percent of fish infected at each sample station, (C) mean parasite richness, and (D) mean number of parasites
In general, our parasitological data agreed with results from diet studies and further confirmed that trophically transmitted parasites were successful in reflecting trophic history and pelagic food web structure in this area of the NCC. The similarities in parasite communities among the fish species examined were similar to results from diet studies on the same fish species within this pelagic food web (Dufault et al. 2009, Miller et al. 2010). In particular, the fish examined in this study were also included in a study by Miller et al. (2010) that examined diet and stable isotopes. Using cluster analysis, their results placed these fish species into similar trophic groups: (a) surf smelt, Pacific sardine, Pacific saury, and northern anchovy (due to dominance of copepods and euphausiid eggs in their diet); (b) juvenile salmonids (due to adult euphausiids and larval-juvenile fish); (c) Pacific hake, Pacific herring, whitebait smelt, and jack mackerel (due to adult euphausiids, decapod larvae, and larval-juvenile fish).

Many of the similarities that we found between parasite communities were due to common trophically transmitted parasites, such as the nematodes Anisakis spp. and Hysterothylacium aduncum. Although Anisakis spp. are generalists, with low specificity for intermediate hosts, euphausiids are the most 
important intermediate hosts for Anisakis spp. (reviewed by Marcogliese 1995). The high prevalence of Anisakis spp. in jack mackerel, Pacific hake, and Pacific herring in our study is in agreement with the high proportion of adult euphausiids in their diet (Dufault et al. 2009, Miller et al. 2010) and the importance of euphausiids in the pelagic food web of the NCC (Dufault et al. 2009). Moreover, Pacific hake that also eat herring (Tanasichuk et al. 1991) likely acquire larval Anisakis spp. by feeding on both infected euphausiids and infected herring. Although the dominance of generalist parasites that use a diversity of zooplankton and fish to complete their life cycles could mask subtle differences in food web structure, parasite species and their abundances were sufficiently different to result in significantly different parasite communities among many host species. However, even with assemblages of only larval stages of generalist parasites, with differences in abundances, it has been possible to determine fish trophic levels in the northern Argentine Sea (Timi et al. 2011) .

\section{Spatial and environmental hypotheses}

Parasite communities of marine fishes are structured by phylogenetic relationships, trophic interactions within a food web, and the environmental conditions of the habitat. Parasites have successfully been used as biological markers of marine fishes because environmental factors can restrict the distribution of 1 or more stages of a life cycle, either directly on a free-living stage or through the distribution of a required host. To date, spatial distributions of marine parasites have been predominantly attributed to large-scale effects, such as latitudinal gradients (Blaylock et al. 1998, González \& Poulin 2005, González et al. 2006). The abiotic drivers of marine parasite distributions are primarily temperature and salinity, acting both directly on free-living stages of marine parasites and on the composition of the invertebrate fauna and ichthyofauna that serve as hosts (see reviews by Polyanski 1961, Marcogliese 2005). In this study, however, neither temperature nor salinity had an effect on the parasite communities of pelagic fishes.

Depth, or distance offshore, can also influence the composition of marine parasite fauna (Polyanski 1961, Marcogliese 2002) and did affect parasite communities in this study. The community structure of trophically transmitted parasites was associated with distance offshore and station depth in June. Distance offshore and station depth are related in this study due to the topography of the coastal shelf, with isobaths running mostly parallel to the coastline. Crossshelf differences were observed in the parasites of jack mackerel in June, with higher intensities of Rhadinorhynchus sp. in jack mackerel caught at offshore stations and higher intensities of Anisakis spp. at inshore stations. We also found high prevalences of Rhadinorhynchus sp., an indicator of offshore fish (George-Nascimento 2000), only in Jack mackerel and Pacific saury, 2 common offshore fish species (Brodeur et al. 2005). These observations are consistent with reports of cross-shelf distribution differences off Oregon in copepod species (Peterson et al. 1979, Morgan et al. 2003, Lamb \& Peterson 2005), euphausiid species and age structure (GómezGutiérrez et al. 2005, Lamb \& Peterson 2005), and ichthyoplankton densities and species compositions (Auth \& Brodeur 2006) that as common intermediate hosts could result in cross-shelf distributions of parasite species. Indeed, the strongest spatial patterns in the distribution of copepod species and community structure are usually zonally (cross-shelf), particularly in upwelling systems, on the order of kilometers; alongshore differences are usually seen only at the $1000 \mathrm{~km}$ scale (Mackas \& Beaugrand 2010). Our observations also support the importance of onshore-offshore gradients in structuring pelagic fish communities in the upwelling regions of the California Current (Brodeur et al. 2005, Harding et al. 2011).

\section{Cape Blanco as a barrier}

Previous studies have suggested that Cape Blanco is a geomorphological feature that affects ecosystem structure in the NCC. Increased wind stress south of the cape results in a broader coastal zone inshore of the upwelling front, a more saline, cooler, denser, and thicker surface mixed layer with higher nutrient concentrations than the upwelling zone to the north of the cape (Huyer et al. 2005). In addition, offshore transport in filaments and jets has been observed at Cape Blanco (Barth et al. 2000). Biologically, higher phytoplankton biomass has been recorded south of the cape (Huyer et al. 2005), and copepod species have been found to differ north and south of the cape (Peterson \& Keister 2002). Offshore transport south of Cape Blanco can have a negative effect on retention of barnacle larvae (Connolly et al. 2001) and fish species (Parrish et al. 1981). Despite these observations on lower trophic level organisms, we found no differences in the parasites of pelagic fish caught north ver- 
sus south of Cape Blanco. For highly migratory species that utilize the entire length of the NCC, such as Pacific hake, jack mackerel, and Pacific sardine, this result is not surprising. However, we found the same result in parasite communities of less migratory species such as Pacific herring and northern anchovy, suggesting that the recorded differences in phytoplankton or zooplankton in the regions north and south of Cape Blanco did not result in differences in the trophic interactions of pelagic fishes in the 2 areas.

Recently, emphasis has been placed on identifying the causes of latitudinal and biogeographical patterns of parasite communities of marine fish by examining the 'decay of similarity' of communities over geographical distance (Oliva \& González 2005, González et al. 2006, 2008, Timi et al. 2010). Decay of similarity can be due to decreasing environmental similarity with distance or a result of geographical barriers that affect the dispersal of organisms (Nekola \& White 1999). Most reports suggest that the contributing factor to decay of similarity for parasite communities of marine fish is a latitudinal gradient of environmental oceanographic conditions, such as temperature (Timi et al. 2010). For instance, González et al. (2008) attributed a distributional break in endoparasites of bigeye flounder Hippoglossina macrops to an upwelling system and a break in the distribution of free-living organisms at $\sim 30^{\circ} \mathrm{S}$ off the coast of Chile associated with a northern warm temperate region and a southern cold temperate region.

A few studies have found differences in marine parasite communities on smaller spatial scales. Vignon et al. (2008) reported differences in the parasite communities of bluestripe snapper Lutjanus kasmira sampled within a few hundred meters between an estuary, lagoon, channel, and outer slope habitats in French Polynesia. Klimpel \& Rükert (2005) found that infections of the nematode Hysterothylacium aduncum were greater in haddock Melanogrammus aeglefinus and whiting Merlangius merlangus caught in highly productive stratified areas in the North Sea (due to high abundances of infected hyperiid amphipods) than in less productive mixed waters. This is the only study we found that directly addressed the influence of oceanic physical systems on the success of a parasite transmission.

In the northern Pacific Ocean, Blaylock et al. (1998) reported an along-shore gradient of parasite distributions for Pacific halibut Hippoglossus stenolepis from Alaska to Washington. On the other hand, parasites of Pacific herring differed on local scales between San Francisco Bay and Tomales Bay, in northern California (Moser \& Hsieh 1992). Beyond these differences observed between parasite communities in separate bays, we have not as yet seen evidence, in our study or others, for differences in parasite communities of marine fishes that result from a geographical barrier that affects the dispersal of marine nekton, such as hypothesized for Cape Blanco.

Although it was proposed that topographic features along the shelf and slope near Cape Blanco cause the upwelling jet to separate from the coast and result in offshore transport of coastal water (Barth et al. 2000), Samelson et al. (2002) showed that enhanced local wind forcing could also contribute to offshore displacement of this upwelling jet. If circulation is more dependent upon persistently strong local winds near and south of the cape than bottom topography, then the separation of the jet may be too episodic to affect the trophic interactions of coastal pelagic fishes and significantly alter transmission rates of trophically transmitted parasites. Our results on the trophically transmitted parasite communities in both June and August, late in the season when the upwelling circulation is fully developed (Barth et al. 2000), support this alternative conclusion. With no persistent separation and only episodic physical effects on zooplankton or high trophic level nekton, Cape Blanco may act as only a periodic geographical barrier for lower trophic marine organisms, with very little effect on the upper trophic food web.

\section{Hotspots}

The transmission of marine parasites is spatially and temporally patchy and may be more likely in geographic areas of high productivity where organisms from several trophic levels converge. Reese \& Brodeur (2006) characterized biological hotspots within the NCC as geographic areas with increased nekton biomass (including zooplankton and fish), abundance, and species richness in relation to specific ranges of environmental variables and suggested that localized biological hotspots are potentially important for migratory species as foraging sites. The 2 identified hotspots had different physical and biological characteristics, but both persisted spatially and temporally. The composition of the fish communities in the hotspots (1 south and 1 north of Cape Blanco) differed with respect to species composition and changed seasonally (Reese \& Brodeur 2006).

Despite the concentration of high nekton abundances in the biological hotspots identified off Hec- 
eta Head, Oregon, and Crescent City, California (Reese \& Brodeur 2006), we found no evidence for higher parasite abundances or diversity in fish caught inside the hotspots compared to outside hotspots in either June or August of 2002. Even with the dominance of generalist parasites in many of the fish species examined, greater abundances of zooplankton and greater foraging would ultimately result in higher abundances of even larval generalist parasites as observed in higher trophic level fishes in the Argentine Sea (Timi et al. 2011). It is possible that fish did not remain in the hotspots long enough to reflect differences through their trophically transmitted parasites. This is supported by the persistence of the hotspots yet changing fish community composition across seasons and years (Reese \& Brodeur 2006). It is also possible that the increases in density and diversity of nekton (zooplankton and fish) in these hotpots were not great enough to increase parasite transmission.

\section{CONCLUSIONS}

The trophically transmitted parasite communities recovered reflected the position of pelagic fish species off of southern Oregon and northern California as well as cross-shelf distributions in zooplankton species or abundances. Ultimately, we were able to use parasite community structure to indicate that oceanographic and biological differences associated with Cape Blanco, or within biological hotspots, were not sufficiently persistent to affect trophic interactions of pelagic fishes in 2002. The trophically transmitted parasite communities of marine fish are an integration of oceanographic conditions and trophic interactions over varying geographic and time scales. Our results suggest that these pelagic fish (even those not highly migratory) do not reside in these local areas long enough to exploit different resources that may be available as a result of oceanographic mesoscale features. Overall, our data suggest that whereas cross-shelf differences in nekton appear to be sufficiently different and persistent to be reflected in marine parasites, temporally the mesoscale features off of southern Oregon may be too episodic to affect pelagic trophic interactions.

Acknowledgements. We thank the captains and crew of the FV 'Sea Eagle' and FV 'Frosti' for their assistance in conducting the trawling operations for these surveys and the numerous members of the Fish Ecology Division of the Northwest Fisheries Science Center, National Marine Fish- eries Service, National Oceanic and Atmospheric Administration, and the Cooperative Institute of Marine Resources Studies staff at Oregon State University that assisted in field sampling and fish necropsies. We also thank a number of internal and journal reviewers for their suggested improvements of this manuscript. Research was conducted as part of the US GLOBEC Northeast Pacific program and was jointly funded by NOAA and the National Science Foundation. This is contribution number 720 of US GLOBEC.

\section{LITERATURE CITED}

Arthur JR, Albert E (1994) A survey of the parasites of Greenland halibut (Reinhardtius hippoglossoides) caught off Atlantic Canada, with notes on their zoogeography in this fish. Can J Zool 72:765-778

Auth TD, Brodeur RD (2006) Distribution and community structure of ichthyoplankton off the coast of Oregon, USA, in 2000 and 2002. Mar Ecol Prog Ser 319:199-213

Bailey KM, Francis RC, Stevens PR (1982) The life history and fishery of Pacific whiting, Merluccius productus. Cal Coop Ocean Fish Invest Rep 23:81-98

Baldwin RE, Miller TW, Brodeur RD, Jacobson K (2008) Expanding foraging history of juvenile Pacific salmon: combining stomach-content and macroparasite-community analyses for studying marine diets. J Fish Biol 72:1268-1294

Barth J, Pierce SD, Smith RL (2000) A separating coastal upwelling jet at Cape Blanco, Oregon and its connection to the California current system. Deep-Sea Res II 47: 783-810

Batchelder HP, Barth JA, Kosro MP, Strub PT and others (2002) The GLOBEC Northeast Pacific California Current System Program. Oceanography 15:36-46

Blaylock R, Margolis L, Holmes JC (1998) Zoogeography of the parasites of Pacific halibut (Hippoglossus stenolepis) in the northeast Pacific. Can J Zool 76:2262-2273

Bray JR, Curtis JT (1957) An ordination of the upland forest communities of southern Wisconsin. Ecol Monogr 27: 325-349

Brodeur RD, Pearcy WG (1992) Effects of environmental variability on trophic interactions and food web structure in a pelagic upwelling system. Mar Ecol Prog Ser 84: 101-119

Brodeur RD, Fisher JP, Teel DJ, Emmett RL, Casillas E, Miller TW (2004) Juvenile salmonid distribution, growth, condition, origin, and environmental and species associations in the Northern California Current. Fish Bull 102:25-46

Brodeur RD, Fisher JP, Emmett RL, Morgan CA, Casillas E (2005) Species composition and community structure of pelagic nekton off Oregon and Washington under variable oceanographic conditions. Mar Ecol Prog Ser 298: 41-57

> Bush AO, Lafferty KD, Lotz JM, Shostak AW (1997) Parasitology meets ecology on its own terms: Margolis et al. revisited. J Parasitol 83:575-583

Campbell RA (1983) Parasitism in the deep sea. In: Rowe GT (ed) The sea, Vol 8. John Wiley \& Sons, New York, NY, p 473-552

Checkley DM, Ayon P, Baumgartner TR, Bernal M and others (2009) Habitats. In: Checkley Jr. D, Alheit J, Oozeki Y, Roy C (eds) Climate change and small pelagic fish. Cambridge University Press, New York, NY, p 12-44 
Clarke KR (1993) Non-parametric multivariate analyses of changes in community structure. Aust J Ecol 18:117-143

- Clarke KR, Ainsworth M (1993) A method of linking multivariate community structure to environmental variables. Mar Ecol Prog Ser 92:205-219

Clarke KR, Gorley RN (2006) Primer v6: user manual/tutorial. Primer-E Ltd., Plymouth Marine Laboratory, Plymouth

Clarke KR, Green RH (1988) Statistical design and analysis for a 'biological effects' study. Mar Ecol Prog Ser 46: 213-226

Clarke KR, Warwick RM (2001) Change in marine communities: an approach to statistical analysis and interpretation, 2nd edn. Primer-E Ltd., Plymouth Marine Laboratory, Plymouth

Connolly SR, Menge BA, Roughgarden J (2001) A latitudinal gradient in recruitment of intertidal invertebrates in the northeast Pacific Ocean. Ecology 82:1799-1831

Dufault AM, Marshall K, Kaplan IC (2009) A synthesis of diets and trophic overlap of marine species in the California Current. US Dept. Commerce, NOAA Tech Memo. NMFS-NWFSC-103

- Emmett RL, Krutzikowsky GH (2008) Nocturnal feeding of Pacific hake and jack mackerel off the mouth of the Columbia River, 1998-2004: implications for juvenile salmon predation. Trans Am Fish Soc 137:657-676

> Flostrand LA, Schweigert JF, Daniel KS, Cleary JS (2009) Measuring and modeling Pacific herring spawning-site fidelity and dispersal using tag-recovery dispersal curves. ICES J Mar Sci 66:1754-1761

Fréon P, Barange M, Arístegui J (2009) Eastern boundary ecosystems: integrative and comparative approaches. Prog Oceanogr 83:1-14

George-Nascimento M (2000) Geographical variations in the jack mackerel Trachurus symmetricus murphyi populations in the southeastern Pacific Ocean as evidenced from the associated parasite communities. J Parasitol 86:929-932

Gómez-Gutiérrez J, Peterson WT, Miller CB (2005) Crossshelf life-stage segregation and community structure of the euphausiids off central Oregon (1970-1972). DeepSea Res II 52:289-315

- González MT, Poulin R (2005) Nested patterns in parasite component communities of a marine fish along its latitudinal range on the Pacific coast of South America. Parasitology 131:569-577

> González MT, Barrientos C, Moreno CA (2006) Biogeographical patterns in endoparasite communities of a marine fish (Sebastes capensis Gmelin) with extended range in the Southern Hemisphere. J Biogeogr 33: 1086-1095

González MT, Vásquez R, Acuña E (2008) Biogeographic patterns of metazoan parasites of the bigeye flounder, Hippoglossina macrops, in the southeastern Pacific coast. J Parasitol 94:429-435

Harding JA, Ammann AJ, MacFarlane RB (2011) Regional and seasonal patterns of epipelagic fish assemblages from the central California Current. Fish Bull 109: 261-281

> Huyer A, Fleischbein JH, Keister J, Kosro PM, Perlin N, Smith RL, Wheeler PA (2005) Two coastal upwelling domains in the northern California Current system. J Mar Res 63:901-929

Keister JE, Cowles TJ, Peterson WT, Morgan CA (2009) Do upwelling filaments result in predictable biological distributions in coastal upwelling ecosystems? Prog Oceanogr 83:303-313

Keister JE, Di Lorenzo E, Morgan CA, Combes V, Peterson WT (2011) Zooplankton species composition is linked to ocean transport in the Northern California Current. Global Change Biol 17:2498-2511

Klimpel S, Seehagen A, Palm HW (2003) Metazoan parasites and feeding behaviour of four small-sized fish species from the central North Sea. Parasitol Res 91:290-297

Klimpel S, Rükert S (2005) Life cycle strategy of Hysterothylacium aduncum to become the most abundant anisakid fish nematode in the North Sea. Parasitol Res 97:141-149

> Lamb J, Peterson W (2005) Ecological zonation of zooplankton in the COAST study region off central Oregon in June and August 2001 with consideration of retention mechanisms. J Geophys Res 110:C10S15 doi:10.1029/ 2004JC002520

- Mackas DL, Beaugrand G (2010) Comparisons of zooplankton time series. J Mar Syst 79:286-304

> Marcogliese DJ (1995) The role of zooplankton in the transmission of helminth parasites to fish. Rev Fish Biol Fish 5:336-371

Marcogliese DJ (2002) Food webs and the transmission of parasites to marine fish. Parasitology 124:S83-S99

Marcogliese DJ (2005) Transmission of marine parasites. In: Rohde K (ed) Marine parasitology. CSIRO Publishing, Canberra, p 280-286

Miller TW, Brodeur RD (2007) Diets of and trophic relationships among dominant marine nekton within the northern California Current ecosystem. Fish Bull 105:548-559

Miller TW, Brodeur RD, Rau G, Omori K (2010) Prey dominance shapes trophic structure of the northern California Current pelagic food web: evidence from stable isotopes and diet analysis. Mar Ecol Prog Ser 420:15-26

Morgan CA, Peterson WT, Emmett RL (2003) Onshore-offshore variations in copepod community structure off the Oregon coast during the summer upwelling season. Mar Ecol Prog Ser 249:223-236

> Moser M, Hsieh J (1992) Biological tags for stock separation in Pacific herring Clupea harengus pallasi in California. J Parasitol 78:54-60

Nekola JC, White PS (1999) The distance decay of similarity in biogeography and ecology. J Biogeogr 26:867-878

> Oliva ME, González MT (2005) The decay of similarity over geographical distance in parasite communities of marine fishes. J Biogeogr 32:1327-1332

Parrish RH, Nelson CS, Bakun N (1981) Transport mechanisms and reproductive success of fishes in the California Current. Biol Oceanogr 1:175-203

Peterson WT, Keister JE (2002) The effect of a large cape on distribution patterns of coastal and oceanic copepods off Oregon and northern California during the 1998-1999 El Niño-La Niña. Prog Oceanogr 53:389-411

> Peterson WT, Miller CB, Hutchinson A (1979) Zonation and maintenance of copepod populations in the Oregon upwelling zone. Deep-Sea Res A 26:467-494

Peterson WT, Brodeur RD, Pearcy WG (1982) Food habits of juvenile salmon in the Oregon coastal zone, June 1979. Fish Bull 80:841-850

> Piatt JF, Wetzel J, Bell K, DeGange AR and others (2006) Predictable hotspots and foraging habitat of the endangered short-tailed albatross (Phoebastria albatrus) in the North Pacific: implications for conservation. Deep-Sea Res II 53:387-398 
Polovina J, Uchida I, Balazs G, Howell EA, Parker D, Button $P$ (2006) The Kuroshio extension bifurcation region: a pelagic hotspot for juvenile loggerhead sea turtles. Deep-Sea Res II 53:326-339

Polyanski YI (1961) Ecology of parasites of marine fishes. In: Dogiel VA, Perushevski GK, Polyanski YI (eds) Parasitology of fishes. Oliver and Boyd Ltd, Edinburgh, p 48-83

Reese DC, Brodeur RD (2006) Identifying and characterizing biological hotspots in the Northern California Current. Deep-Sea Res II 53:291-314

Rohde K (1984) Ecology of marine parasites. Helgol Meersunters 37:5-33

Samelson R, Barbour P, Barth J, Bielli S and others (2002) Wind stress forcing of the Oregon coastal ocean during the 1999 upwelling season. J Geophys Res 107:3034 doi:10.1029/2001JC000900

Schabetsberger R, Morgan CA, Brodeur RD, Potts CJ, Peterson WT, Emmett RL (2003) Prey selectivity and diel feeding chronology of juvenile chinook (Oncorhynchus tshawytscha) and coho (O. kisutch) salmon in the Columbia River plume. Fish Oceanogr 12:523-540

Smith JW (1974) Experimental transfer of Anisakis sp. larvae (Nematoda: Ascaridida) from one fish host to another. J Helminthol 48:229-234

Strub PT, Batchelder HP, Weingartner TJ (2002) U.S. GLOBEC Northeast Pacific Program: overview. Oceanography 15:30-35

Sydeman WJ, Brodeur RD, Grimes CB, Bychkov AS, McKinnell S (2006) Marine habitat 'hotspots' and their use by migratory species and top predators in the north Pacific Ocean: introduction. Deep-Sea Res II 53:247-249

Editorial responsibility: Stylianos Somarakis, Heraklion, Greece
Tanasichuk RW, Ware DM, Shaw W, McFarlane GA (1991) Variations in the diet, daily ration and feeding periodicity of Pacific hake (Merluccius productus) and spiny dogfish (Squalus acanthias) off the lower west coast of Vancouver Island. Can J Fish Aquat Sci 48:2118-2128

Thompson RM, Mouritsen KN, Poulin R (2005) Importance of parasites and their life cycle characteristics in determining the structure of a large marine food web. J Anim Ecol 74:77-85

Timi JT, Lanfranchi AL, Luque JL (2010) Similarity in parasite communities of the teleost fish Pinguipes brasilianus in the southwestern Atlantic: infracommunities as a tool to detect geographical patterns. Int $\mathrm{J}$ Parasitol 40: 243-254

Timi JT, Rossin MA, Alarcos AJ, Braicovich PE, Cantatore DMP, Lanfaranchi AL (2011) Fish trophic level and the similarity of non-specific larval parasite assemblages. Int J Parasitol 41:309-316

Valavanis VD, Kapantagakis A, Katara I, Palialexis A (2004) Critical regions: a GIS-based model of marine productivity hotspots. Aquat Sci 66:139-148

> Valtonen ET, Marcogliese DJ, Julkunen M (2010) Vertebrate diets derived from trophically transmitted fish parasites in the Bothnian Bay. Oecologia 162:139-152

van der Lingen C, Bertrand A, Bode A, Brodeur R and others (2009) Trophic dynamics. In: Checkly D, Alheit J, Oozeki Y, Claude R (eds) Climate change and small pelagic fish. Cambridge University Press, Cambridge, p 112-157

Vignon M, Morat F, Galzin R, Sasal P (2008) Evidence for spatial limitation of the bluestripe snapper Lutjanus kasmira in French Polynesia from parasite and otolith shape analysis. J Fish Biol 73:2305-2320

Submitted: August 2, 2011; Accepted: February 8, 2012

Proofs received from author(s): April 27, 2012 